\title{
Tests of analytical hadronisation models using event shape moments in $\mathrm{e}^{+} \mathrm{e}^{-}$annihilation
}

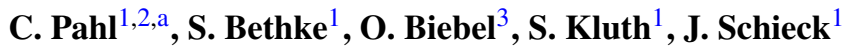 \\ ${ }^{1}$ Max-Planck-Institut für Physik, Föhringer Ring 6, 80805 Munich, Germany \\ ${ }^{2}$ Excellence Cluster Universe, Technische Universität München, Boltzmannstr. 2, 85748 Garching, Germany \\ ${ }^{3}$ LMU München, Fakultät für Physik, Am Coulombwall 1, 85748 Garching, Germany
}

Received: 31 March 2009 / Revised: 29 July 2009 / Published online: 20 October 2009

(C) Springer-Verlag / Società Italiana di Fisica 2009

\begin{abstract}
Predictions of analytical models for hadronisation, namely the dispersive model, the shape function and the single dressed gluon approximation, are compared with moments of hadronic event shape distributions measured in $\mathrm{e}^{+} \mathrm{e}^{-}$annihilation at centre-of-mass energies between 14 and $209 \mathrm{GeV}$. In contrast to Monte Carlo models for hadronisation, analytical models require to adjust only two universal parameters, the strong coupling and a second quantity parametrising non-perturbative corrections. The extracted values of $\alpha_{\mathrm{S}}$ are consistent with the world average and competitive with previous measurements. The variance of event shape distributions is compared with predictions given by some of these models. Limitations of the models, probably due to unknown higher order corrections, are demonstrated and discussed.
\end{abstract}

PACS 12.38.Lg $\cdot$ 12.38.Qk

\section{Introduction}

In previous studies, moments of event shape distributions have been compared to perturbative predictions of quantum chromodynamics (QCD, [1-4]) in next-to-leading order, simulating the hadronisation process by Monte Carlo models [5, 6]. Alternatively there exist models describing hadronisation analytically. This paper aims to study these models qualitatively and quantitatively by measurements of the strong coupling and the model parameters.

The dispersive model [7] is based on the assumption of a non-perturbatively continued strong coupling. The shape function [8] additionally describes a modification of the shape of the perturbatively calculated distribution. The single dressed gluon approximation [9, 10] estimates the per-

a e-mail: pahl@mppmu.mpg.de turbative part more completely with reduced perturbative uncertainties of the prediction. The models test the predicted energy evolution of the strong coupling. Their parameters can be determined-one of them the value of $\alpha_{\mathrm{S}}$ at some reference energy - and the assumption of universality of these parameters can be probed. To date, primarily the distributions themselves and the mean values (first moments) have been studied [11-14].

This analysis uses data measured by JADE [5] in the years $1979 \ldots 1986$ at six centre-of-mass (cms) energies in the energy range of $Q=14 \ldots 44 \mathrm{GeV}$, and data measured by OPAL at 12 energy points over the whole LEP energy range of $91 \ldots 209 \mathrm{GeV}$ and combined into 4 energy ranges [6]. The large energy range covered by the measurements allows to test the employed assumptions selectively.

The outline of the paper is as follows. In Sect. 2, we present the observables used in the analysis. In Sect. 3 we describe the perturbative QCD predictions and introduce the analytical models which describe the hadronisation process. In Sect. 4 we discuss predictions for event shape moments at hadron level and compare them with the measurements. The predictions for the event shape distribution variance following from the dispersive model and the shape function are tested as well. In Sect. 5 we summarize and give our conclusions.

\section{Event shape moments}

Event shape variables are a convenient way to characterise properties of hadronic final states. They are calculated from particle momenta and energies. For definitions of the variables we refer to [6].

In a hadronic event in $\mathrm{e}^{+} \mathrm{e}^{-}$annihilation the virtual vector boson $Z^{0} / \gamma^{*}$ generated by annihilation of electron and positron decays into a quark pair $\mathrm{q} \overline{\mathrm{q}}$. The quarks may radi- 
ate gluons which radiate further gluons or decay into another quark pair. The final state of this parton shower is called parton level. By the process of hadronisation partons are transferred into hadrons. The predictions used in this work describe the hadron level. The variables measured in the experiments have to be corrected for the effects of limited detector acceptance and resolution to probe the hadron level.

The event shapes considered here are thrust $T$, Cparameter $C$, heavy jet mass $M_{\mathrm{H}}$, jet broadening variables $B_{\mathrm{T}}$ and $B_{\mathrm{W}}$, and the transition value $y_{23}^{\mathrm{D}}$ between 2- and 3jet final states defined using the Durham jet reconstruction scheme. The $\alpha_{S}$ determination in [5] is based on moments of these variables, in [6] on distributions and moments of these variables. Their theoretical description by perturbation theory is currently the most advanced [15-17].

A generic event shape variable is denoted by the symbol $y$. Regions dominated by multiple jets give large values of $y$, while two narrow jets give $y \simeq 0$. Thrust $T$ is an exception to this rule. By using $y=1-T$ instead, the condition is fulfilled for all event shapes. $B_{\mathrm{W}}, y_{23}^{\mathrm{D}}$ and $M_{\mathrm{H}}$ are sensitive to only one suitably chosen hemisphere of the event (one-hemisphere variables), $1-T, C$ and $B_{\mathrm{T}}$ are sensitive to the whole hadronic event (two-hemisphere variables).

The $n$ th, $n=1,2, \ldots$ moment of the distribution of an event shape variable $y$ is defined by

$\left\langle y^{n}\right\rangle=\int_{0}^{y_{\max }} \mathrm{d} y y^{n} \frac{1}{\sigma_{\text {tot. }}} \frac{\mathrm{d} \sigma}{\mathrm{d} y}$,

where $y_{\max }$ is the kinematically allowed upper limit of the variable and $\sigma_{\text {tot. }}$ denotes the total hadronic cross section.

Predictions have been made available for the moments of event shapes. Their evolution with cms energy allows direct tests of the predicted energy evolution of the strong coupling $\alpha_{\mathrm{S}}$. Furthermore it enables the determination of a single value of $\alpha_{S}$ at a definite energy scale-for example $\alpha_{\mathrm{S}}\left(M_{\mathrm{Z}^{0}}\right)$ at the rest energy of the $\mathrm{Z}^{0}$ boson. The theoretical calculations always involve a full integration over phase space, which implies that comparison with data always probes all of the available phase space. This is in contrast to QCD predictions for distributions; these are commonly only compared with data-e.g. in order to measure $\alpha_{S}$-in restricted regions, where the theory is able to describe the data well, see e.g. [18]. Comparisons of QCD predictions for moments of event shape distributions with data are thus complementary to tests of the theory using distributions.

\section{Theory}

The QCD prediction for $\left\langle y^{n}\right\rangle$ in next-to-leading-order ${ }^{1}$ (NLO) of the strong coupling $\bar{\alpha}_{\mathrm{S}} \equiv \alpha_{\mathrm{S}} /(2 \pi)$ has the form ${ }^{2}$

$\left\langle y^{n}\right\rangle_{\mathrm{NLO}}=\mathcal{A}_{n} \bar{\alpha}_{\mathrm{S}}+\left(\mathcal{B}_{n}-2 \mathcal{A}_{n}\right) \bar{\alpha}_{\mathrm{S}}^{2}$

The coefficients $\mathcal{A}_{n}$ and $\mathcal{B}_{n}$ are obtained by numerical integration of the QCD matrix elements using the program EVENT2 [20]. These predictions were also used in [5, 6].

The coupling $\bar{\alpha}_{\mathrm{S}}$, and therefore the QCD prediction depends on the renormalisation scale $\mu_{R}$, see e.g. [21]. The prediction is changed by this dependence as shown in [5]. For clearer notation the renormalisation scale factor is defined as $x_{\mu} \equiv \mu_{R} / Q$; a truncated fixed order QCD calculation such as (2) will then depend on $x_{\mu}$.

Infrared and collinear stability are essential for a perturbative description of the parton level. These properties, however, do not suffice for a perturbative description of the hadron level as hadronisation takes place at low energy scales, where the perturbative description breaks down. The evolution of partons to hadrons can be approximated by analytical calculations. These analytical calculations are generally motivated from the transition from perturbative to nonperturbative regime. A perturbation series in quantum field theory is a divergent series, see e.g. [22]. To obtain finite results, regularisations have to be applied. From suitable prescriptions non-perturbative terms are found, which typically scale with inverse powers of the cms energy [7-9].

\subsection{Dispersive model}

This model $[7,24,25]$ is based on the assumption of a non-perturbatively defined strong coupling $\alpha_{\mathrm{S}}\left(Q^{2}\right)$ which remains finite at and below the Landau pole $\Lambda_{\mathrm{QCD}}$. The Landau pole is the scale where the usual perturbative coupling diverges. The matching scale $Q=\mu_{I}$, marking the border between perturbative and non-perturbative region, is not uniquely defined. Usually it is taken as $\mu_{I} \simeq 2 \mathrm{GeV}$.

As the non-perturbative coupling cannot be calculated, it is parametrised universally in a simple way by the zeroth moment of the extended coupling over the non-perturbative region,

$\alpha_{0}\left(\mu_{I}\right)=\frac{1}{\mu_{I}} \int_{0}^{\mu_{I}} \mathrm{~d} k \alpha_{S}\left(k^{2}\right)$.

\footnotetext{
${ }^{1}$ Very recently, perturbative NNLO predictions for event shape moments became available [19]. The non perturbative predictions have to be adapted, and comparing to NNLO is beyond the scope of this work. ${ }^{2}$ The $\bar{\alpha}_{\mathrm{S}}^{2}$ coefficient is written as $\mathcal{B}_{n}-2 \mathcal{A}_{n}$ because the QCD calculations are normalized to the Born cross section $\sigma_{0}$, while the data are normalized to the total hadronic cross section $\sigma_{\text {tot. }}=\sigma_{0}\left(1+2 \bar{\alpha}_{\mathrm{S}}\right)$ in LO.
} 
In first approximation, non-perturbative corrections generate a simple shift of the perturbative differential distribution $\mathrm{d} \sigma_{\mathrm{NLO}} / \mathrm{d} y$ of the event shape variables $1-T, C, B_{\mathrm{T}}, B_{\mathrm{W}}$ and $^{3} M_{\mathrm{H}}^{2}$ when relating parton to hadron level,

$\frac{\mathrm{d} \sigma_{\mathrm{had}}}{\mathrm{d} y}=\frac{\mathrm{d} \sigma_{\mathrm{NLO}}}{\mathrm{d} y}\left(y-a_{y} \cdot \mathcal{P}\right)$

This prediction is valid only if the value of the event shape variable $y$ is not too large $(y \ll 1)$, and the cms energy $Q$ not too small $\left(Q \gg \Lambda_{\mathrm{QCD}} / y\right)$. Only the numerical factor $a_{y}$ depends on the event shape variable $y$, see Table 1 . However $\mathcal{P}$ depends on the hard scale $Q$ but is universal for the event shape variables $1-T, C, B_{\mathrm{T}}, B_{\mathrm{W}}$ and $M_{\mathrm{H}}^{2}$, and has the form $[24,25]$

$$
\begin{aligned}
\mathcal{P}= & \frac{4 C_{F}}{\pi^{2}} \cdot \mathcal{M} \cdot\left\{\alpha_{0}\left(\mu_{I}\right)-\left[\alpha_{\mathrm{S}}\left(\mu_{R}^{2}\right)\right.\right. \\
& \left.\left.+2 \beta_{0} \alpha_{\mathrm{S}}^{2}\left(\mu_{R}^{2}\right)\left(\ln \frac{\mu_{R}}{\mu_{I}}+1+\frac{K}{4 \pi \beta_{0}}\right)+\mathcal{O}\left(\alpha_{\mathrm{S}}^{3}\right)\right]\right\} \\
& \times \frac{\mu_{I}}{Q} .
\end{aligned}
$$

In the $\overline{\mathrm{MS}}$ renormalisation scheme the constant $K$ has the value

$K=C_{A}\left(\frac{67}{18}-\frac{\pi^{2}}{6}\right)-\frac{5}{9} n_{\mathrm{f}}$

with $n_{\mathrm{f}}=5$ at the studied energies, and the beta function coefficient is $\beta_{0}=23 /(12 \pi)$. The color factors have the values $C_{F}=4 / 3, C_{A}=3$ [26], the Milan factor $\mathcal{M}$ is known in two loops, $\mathcal{M}=1.49 \pm 20 \%$ [27-29] (for flavour number $n_{\mathrm{f}}=3$ at the relevant low scales). The cited uncertainty results [25] from the estimation of the next order contribution, $\mathcal{M}_{\mathrm{NNLO}}=\mathcal{M}_{\mathrm{NLO}} \cdot\left(1+\mathcal{O}\left(\alpha_{\mathrm{S}} / \pi\right)\right)$.

Applying the dispersive model calculation (4) of the normalized event shape distribution in definition (1) of the moment of order $n$ and naively neglecting the integration over the unphysical range of negative variable values, gives

$\left\langle y^{n}\right\rangle=\int_{0}^{1} \mathrm{~d} y y^{n} \cdot \frac{\mathrm{d} \sigma}{\mathrm{d} y}(y) \approx \int_{0}^{1} \mathrm{~d} y\left(y+a_{y} \mathcal{P}\right)^{n} \cdot \frac{\mathrm{d} \sigma_{\mathrm{NLO}}}{\mathrm{d} y}(y)$.

Table 1 Coefficients $a_{y}$ of power correction $\propto 1 / Q$ of event shape variables in the dispersive model [27, 28]

\begin{tabular}{llllll}
\hline $\begin{array}{l}\text { Event shape } \\
\text { variable } y\end{array}$ & $1-T$ & $C$ & $B_{\mathrm{T}}$ & $B_{\mathrm{W}}$ & $M_{\mathrm{H}}^{2}$ \\
\hline$a_{y}$ & 2 & $3 \pi$ & 1 & $1 / 2$ & 1 \\
\hline
\end{tabular}

${ }^{3}$ The theoretical calculations are based on the variable $M_{\mathrm{H}}^{2}$ because of the problems with the NLO description of $M_{\mathrm{H}}$ as discussed in [5, 6, 23].
The predictions for the moments on hadron level become:

$$
\begin{aligned}
\left\langle y^{1}\right\rangle= & \left\langle y^{1}\right\rangle_{\mathrm{NLO}}+a_{y} \mathcal{P} \\
\left\langle y^{2}\right\rangle= & \left\langle y^{2}\right\rangle_{\mathrm{NLO}}+2\left\langle y^{1}\right\rangle_{\mathrm{NLO}} \cdot a_{y} \mathcal{P}+\left(a_{y} \mathcal{P}\right)^{2} \\
\left\langle y^{3}\right\rangle= & \left\langle y^{3}\right\rangle_{\mathrm{NLO}}+3\left\langle y^{2}\right\rangle_{\mathrm{NLO}} \cdot a_{y} \mathcal{P}+3\left\langle y^{1}\right\rangle_{\mathrm{NLO}} \cdot\left(a_{y} \mathcal{P}\right)^{2} \\
& +\left(a_{y} \mathcal{P}\right)^{3}, \\
\left\langle y^{4}\right\rangle= & \left\langle y^{4}\right\rangle_{\mathrm{NLO}}+4\left\langle y^{3}\right\rangle_{\mathrm{NLO}} \cdot a_{y} \mathcal{P}+6\left\langle y^{2}\right\rangle_{\mathrm{NLO}} \cdot\left(a_{y} \mathcal{P}\right)^{2} \\
& +4\left\langle y^{1}\right\rangle_{\mathrm{NLO}} \cdot\left(a_{y} \mathcal{P}\right)^{3}\left(a_{y} \mathcal{P}\right)^{4}, \\
\left\langle y^{5}\right\rangle= & \left\langle y^{5}\right\rangle_{\mathrm{NLO}}+5\left\langle y^{4}\right\rangle_{\mathrm{NLO}} \cdot a_{y} \mathcal{P}+10\left\langle y^{3}\right\rangle_{\mathrm{NLO}} \cdot\left(a_{y} \mathcal{P}\right)^{2} \\
& +10\left\langle y^{2}\right\rangle_{\mathrm{NLO}} \cdot\left(a_{y} \mathcal{P}\right)^{3}+5\left\langle y^{1}\right\rangle_{\mathrm{NLO}} \cdot\left(a_{y} \mathcal{P}\right)^{4} \\
& +\left(a_{y} \mathcal{P}\right)^{5} .
\end{aligned}
$$

Previous studies [30] indicate that the parameters $\alpha_{\mathrm{S}}\left(M_{\mathrm{Z}^{0}}\right)$ and $\alpha_{0}(2 \mathrm{GeV})$ when fitted to $B_{\mathrm{T}}$ and $B_{\mathrm{W}}$ distributions via (4) are not compatible with values derived from $1-T$ and $C$. Therefore improved predictions for these distributions were given. They also describe a compression of the distribution peak, i.e. a narrowing in the two jet region $[25,31]$. The non-perturbative factor $\mathcal{P}$ in the case of $\left\langle B_{\mathrm{T}}^{1}\right\rangle$ and $\left\langle B_{\mathrm{W}}^{1}\right\rangle$ is replaced by [32]

$$
\begin{aligned}
\mathcal{P}_{\left\langle B_{\mathrm{T}}^{1}\right\rangle}= & \mathcal{P} \cdot\left(\frac{\pi}{2 \sqrt{C_{F} \widehat{\alpha}_{S}\left(1+K \widehat{\alpha}_{s} /(2 \pi)\right)}}\right. \\
& \left.+\frac{3}{4}-\frac{2 \pi \beta_{0}}{3 C_{F}}+\eta_{0}\right)
\end{aligned}
$$

rsp.

$$
\begin{aligned}
\mathcal{P}_{\left\langle B_{\mathrm{W}}^{1}\right\rangle}= & \mathcal{P} \cdot\left(\frac{\pi}{2 \sqrt{2 C_{F} \widehat{\alpha}_{S}\left(1+K \widehat{\alpha}_{S} /(2 \pi)\right)}}\right. \\
& \left.+\frac{3}{4}-\frac{\pi \beta_{0}}{3 C_{F}}+\eta_{0}\right),
\end{aligned}
$$

with a rescaled coupling $\widehat{\alpha}_{S}\left(Q^{2}\right) \equiv \alpha_{\mathrm{S}}\left(\mathrm{e}^{-3 / 2} Q^{2}\right)$ and a constant $\eta_{0} \simeq-0.6137$.

No power correction coefficient has been calculated in the dispersive model for the variable $y_{23}^{\mathrm{D}}$. The purely perturbative prediction describes the first moments of $y_{23}^{\mathrm{D}}$ well [14, 32 _ - therefore we also compare it with the higher moments.

The dispersive model gives predictions for several observables and contains only universal free parameters $\alpha_{\mathrm{S}}\left(M_{\mathrm{Z}^{0}}\right)$ and $\alpha_{0}\left(\mu_{I}\right)$.

\subsection{Shape function}

Korchemsky and Tafat [8] describe properties of the event shape variables $1-T, C$ and $M_{\mathrm{H}}^{2}$ not included in NLO perturbation theory by a so called shape function, which does not depend on the variable nor the cms energy. This is more 
general than the dispersive model, as not only a shift of the perturbative prediction is predicted but also a compression of the distribution peak.

The prediction is deduced from studying the two jet region (i.e. $y \ll 1$ ) in the distribution of the event shape variable $y$. The prediction for the differential distribution is

$\frac{1}{\sigma} \frac{\mathrm{d} \sigma(Q)}{\mathrm{d} y}=\int_{0}^{Q \cdot y} \mathrm{~d} \varepsilon f_{y}(\varepsilon) \frac{\mathrm{d} \sigma_{\mathrm{NLO}}}{\mathrm{d} y}(y-\varepsilon / Q)$,

with a non-perturbative function $f_{y}(\varepsilon)$, dependent on one scale parameter $\varepsilon$. This function is derived from the shape function $f\left(\varepsilon_{L}, \varepsilon_{R}\right)$ [8], which depends on two scale parameters $\varepsilon_{L}, \varepsilon_{R}$ for the two hemispheres of the event. By the compression of the distribution the validity of the prediction is extended compared to the dispersive model to $y \simeq \Lambda_{\mathrm{QCD}} / Q$.

The shape function can be parametrised [8] by its "first moment",

$\lambda_{1}=\int \mathrm{d} \varepsilon_{R} \int \mathrm{d} \varepsilon_{L}\left(\varepsilon_{R}+\varepsilon_{L}\right) f\left(\varepsilon_{R}, \varepsilon_{L}\right) \equiv\left\langle\varepsilon_{R}+\varepsilon_{L}\right\rangle$,

its "second moment",

$\lambda_{2}=\left\langle\left(\varepsilon_{R}+\varepsilon_{L}\right)^{2}\right\rangle$,

and a $Q$ dependent function $\delta \lambda_{2}(Q)$. Predictions for the moments of event shape variables can be derived, and $\lambda_{1}$ and $\lambda_{2}$ can be fitted to the data.

From prediction (14) for the distribution of the variables $1-T, C$ and $M_{\mathrm{H}}^{2}$, predictions for the mean values follow [8] by integration

$$
\begin{aligned}
\left\langle(1-T)^{1}\right\rangle & =\left\langle(1-T)^{1}\right\rangle_{\mathrm{NLO}}+\frac{\lambda_{1}}{Q} \\
\left\langle C^{1}\right\rangle & =\left\langle C^{1}\right\rangle_{\mathrm{NLO}}+\frac{3 \pi}{2} \frac{\lambda_{1}}{Q}\left[1-5.73 \frac{\alpha_{s}\left(Q^{2}\right)}{2 \pi}\right] \\
\left\langle M_{H}^{2}\right\rangle & =\left\langle M_{H}^{2}\right\rangle_{\mathrm{NLO}}+\frac{\lambda_{1}}{2 Q} .
\end{aligned}
$$

Analogously for the second moments one finds [8],

$$
\begin{aligned}
\left\langle(1-T)^{2}\right\rangle= & \left\langle(1-T)^{2}\right\rangle_{\mathrm{NLO}}+2 \frac{\lambda_{1}}{Q}\langle 1-T\rangle_{\mathrm{NLO}}+\frac{\lambda_{2}}{Q^{2}}, \quad \text { (20) } \\
\left\langle C^{2}\right\rangle= & \left\langle C^{2}\right\rangle_{\mathrm{NLO}}+\frac{3 \pi}{2} \frac{\lambda_{1}}{Q}\left[2\langle C\rangle_{\mathrm{NLO}}-4.30 \frac{\alpha_{\mathrm{S}}\left(Q^{2}\right)}{2 \pi}\right] \\
& +\frac{9 \pi^{2}}{4} \frac{\lambda_{2}}{Q^{2}}\left[1-11.46 \frac{\alpha_{\mathrm{S}}\left(Q^{2}\right)}{2 \pi}\right], \\
\left\langle M_{\mathrm{H}}^{4}\right\rangle= & \left\langle M_{\mathrm{H}}^{4}\right\rangle_{\mathrm{NLO}}+\frac{\lambda_{1}}{Q}\left\langle M_{H}^{2}\right\rangle_{\mathrm{NLO}}+\frac{\lambda_{2}+\delta \lambda_{2}(Q)}{4 Q^{2}} .
\end{aligned}
$$

In this model the more strongly suppressed power corrections have an independent coefficient. The coefficient $\lambda_{1}$ is interpreted as the first "moment" of the shape function, $\lambda_{2}$ the second, and $\delta \lambda_{2}$ as a contribution accounting for the onehemisphere character of $M_{\mathrm{H}}^{2}$. Therefore these are universal scales.

\subsection{Variance of event shape distributions}

A theory predicting first and second moments of an event shape variable $y$ also gives a prediction for the variance $\operatorname{Var}(y)=\left\langle y^{2}\right\rangle-\langle y\rangle^{2}$. In [33] the variance of event shape distributions on hadron level is calculated in the dispersive model. Power corrections suppressed less than $\alpha_{\mathrm{S}} / Q^{2}$ are found to cancel. So the variance is described in the dispersive model perturbatively without significant power corrections. This would open the possibility of an accurate $\alpha_{\mathrm{S}}\left(M_{\mathrm{Z}^{0}}\right)$ determination.

This simple prediction for the variance of event shape variables on hadron level is deduced [33] by employing the predictions (7) and (8),

$\operatorname{Var}(y)=\left\langle y^{2}\right\rangle_{\mathrm{NLO}}-\langle y\rangle_{\mathrm{NLO}}^{2}$.

In the dispersive model one obtains a purely perturbative expression for the variance, up to strongly suppressed corrections $\mathcal{O}\left(\alpha_{\mathrm{S}} / Q^{2}\right)$.

Predictions for the event shape variance can also be derived from the shape function. The first and second moment predictions (17) and (20) give the identical prediction in case of thrust,

$\operatorname{Var}(1-T)=\left\langle(1-T)^{2}\right\rangle_{\mathrm{NLO}}-\langle 1-T\rangle_{\mathrm{NLO}}^{2}$.

Analogously this follows from (19) and (22) for $\operatorname{Var}\left(M_{\mathrm{H}}^{2}\right)$. $\operatorname{Var}(C)$ follows from (18), (21) and the perturbative LO coefficient [20]; up to $\mathcal{O}\left(1 / Q^{2}\right)$

$\operatorname{Var}(C)=\left\langle C^{2}\right\rangle_{\mathrm{NLO}}-\langle C\rangle_{\mathrm{NLO}}^{2}-3.23 \frac{\lambda_{1}}{Q} \alpha_{s}\left(Q^{2}\right)$,

showing an additional term $\propto \alpha_{\mathrm{S}} / Q$ with coefficient $\lambda_{1}$ as in Sect. 3.2.

\subsection{Single dressed gluon approximation}

Gardi et al. [9, 10] assume the existence of a reordering of the perturbative series, the so called skeleton expansion [34] - its existence is proven only for abelian field theory like QED. The first contribution to this expansion is a single dressed gluon (SDG) which resums running coupling effects of any order in $\alpha_{\mathrm{S}}$. These are renormalons in the dominant contributions $\propto \beta_{0}^{n}$. The single dressed gluon graphs can be calculated completely. In this way the perturbative prediction $\left\langle(1-T)^{n}\right\rangle_{\mathrm{pt}}$. for a moment of thrust can be approximated. This approximated series already diverges and 
various regularisations differ by definite powers of the $\mathrm{cms}$ energy $Q$. These predictions follow:

$$
\begin{aligned}
& \left\langle(1-T)^{1}\right\rangle=\left\langle(1-T)^{1}\right\rangle_{\text {pt. }}+\frac{\nu_{1}}{Q}, \\
& \left\langle(1-T)^{2}\right\rangle=\left\langle(1-T)^{2}\right\rangle_{\text {pt. }}+\frac{\nu_{2}}{Q^{2}}+\frac{\kappa_{2}}{Q^{3}}, \\
& \left\langle(1-T)^{3}\right\rangle=\left\langle(1-T)^{3}\right\rangle_{\text {pt. }}+\frac{\nu_{3}}{Q^{2}}+\frac{\kappa_{3}}{Q^{3}}, \\
& \left\langle(1-T)^{4}\right\rangle=\left\langle(1-T)^{4}\right\rangle_{\text {pt. }}+\frac{\nu_{4}}{Q^{2}}+\frac{\kappa_{4}}{Q^{5}} .
\end{aligned}
$$

In general, the non-perturbative correction is predicted as a sum of two terms with different powers in $Q$ and coefficients $v_{n}, \kappa_{n}$.

The thrust is used in the massless limit,

$$
T=\frac{\sum_{i}\left|\boldsymbol{p}_{i} \cdot \boldsymbol{n}_{T}\right|}{\sum_{i} E_{i}}=\frac{\sum_{i}\left|\boldsymbol{p}_{i} \cdot \boldsymbol{n}_{T}\right|}{Q} .
$$

Here the denominator is changed with respect to the standard definition [6] by $\sum_{i}\left|\boldsymbol{p}_{i}\right| \mapsto \sum_{i} E_{i}$, which does not change the thrust value as long as massless partons are considered. Under this change of definition the thrust value calculated with a "massive" virtual gluon is correct, as long as all (massless) partons generated in the hadronisation end up in the same event hemisphere w.r.t. to the thrust axis. This so called "inclusive" calculation is valid only if fragmentation of the gluon is approximately collinear. The perturbative SDG predictions for the moments of thrust are given as perturbation series in the coupling $\bar{a}$ [10], which relates to $\alpha_{\mathrm{S}}\left(\mu_{R}^{2}\right)$ in the $\overline{\mathrm{MS}}$ scheme by a simple shift of the Landau Pole, ${ }^{4}$

$\bar{a}\left(\mu_{R}^{2}\right) \equiv \frac{\alpha_{\mathrm{S}}\left(\mu_{R}^{2}\right) / \pi}{1-\frac{5}{3} \beta_{0} \alpha_{\mathrm{S}}\left(\mu_{R}^{2}\right)}$.

The perturbative coefficients are given in so called $\log$ moments $d_{i}$ of the characteristic thrust function. The first six $\log$ moments for $\left\langle(1-T)^{1}\right\rangle \ldots\left\langle(1-T)^{4}\right\rangle$ were calculated by numerical integration [10]. The perturbative prediction of $\mathcal{O}\left(\bar{\alpha}_{\mathrm{S}}^{6}\right)$ in the (obviously incomplete) SDG approximation has the form [35]

$$
\begin{aligned}
\left\langle(1-T)^{n}\right\rangle_{\mathrm{SDG}} \\
=A_{n} \cdot \bar{a}+B_{n} \cdot \bar{a}^{2}+C_{n} \cdot \bar{a}^{3}+D_{n} \cdot \bar{a}^{4}+E_{n} \cdot \bar{a}^{5} \\
\quad+F_{n} \cdot \bar{a}^{6} \\
=d_{0} \cdot \bar{a}+\beta_{0} d_{1} \pi \cdot \bar{a}^{2} \\
\quad+\left(\left(-\frac{1}{3} d_{0} \pi^{2}+d_{2}\right) \beta_{0}^{2}+\beta_{1} d_{1}\right) \pi^{2} \cdot \bar{a}^{3}
\end{aligned}
$$

${ }^{4}$ In particular the beta function coefficients $\beta_{i}$ have the same values as in the $\overline{\mathrm{MS}}$ scheme.

$$
\begin{aligned}
& +\left(\left(-\pi^{2} d_{1}+d_{3}\right) \beta_{0}^{3}+\frac{5}{2} \beta_{1} \beta_{0} d_{2}+\beta_{2} d_{1}\right) \pi^{3} \cdot \bar{a}^{4} \\
& +\left(\left(d_{4}+\frac{1}{5} d_{0} \pi^{4}-2 \pi^{2} d_{2}\right) \beta_{0}^{4}\right. \\
& +\left(\frac{13}{3} \beta_{1} d_{3}-\beta_{1} \pi^{2} d_{1}\right) \beta_{0}^{2} \\
& \left.+3 \beta_{2} d_{2} \beta_{0}+\frac{3}{2} \beta_{1}^{2} d_{2}+\beta_{3} d_{1}\right) \pi^{4} \cdot \bar{a}^{5} \\
& +\left(\left(-\frac{10}{3} \pi^{2} d_{3}+\pi^{4} d_{1}+d_{5}\right) \beta_{0}^{5}\right. \\
& +\left(\frac{77}{12} d_{4}-\frac{9}{2} \pi^{2} d_{2}\right) \beta_{1} \beta_{0}^{3} \\
& +\left(6 d_{3}-\pi^{2} d_{1}\right) \beta_{2} \beta_{0}^{2}+\left(\frac{35}{6} \beta_{1}^{2} d_{3}+\frac{7}{2} \beta_{3} d_{2}\right) \beta_{0} \\
& \left.+\beta_{4} d_{1}+\frac{7}{2} \beta_{1} \beta_{2} d_{2}\right) \pi^{5} \cdot \bar{a}^{6},
\end{aligned}
$$

where the $n$-dependent $d_{i}$ are taken from [10]. Higher order contributions from the running of the coupling are accounted for up to sixth order. Reference [10] gives the prediction explicitly in $\mathcal{O}\left(\bar{a}^{3}\right)$ without the term $\beta_{1} d_{1} \pi^{2} \cdot \bar{a}^{3}$. This term with marked effect on the $\bar{a}^{3}$-coefficient results from considering the usual QCD beta function [35]. The symbols $\beta_{0} \ldots \beta_{3}$ denote the usual beta function coefficients [36, 37], $\beta_{4}$ is unknown. We set $\beta_{4}$ to 0 .

The SDG approximation (27) is complete in leading or$\operatorname{der} \mathcal{O}(\bar{a})$ by construction [10]. In higher orders this approximation only gives the terms $\propto \beta_{0}$. Therefore we use the numerically calculated [20] NLO coefficients. For the third and higher orders we employ the SDG approximation,

$$
\begin{aligned}
& \left\langle(1-T)^{n}\right\rangle_{\mathrm{pt.}} \\
& =\left\langle(1-T)^{n}\right\rangle_{\mathrm{NLO}} \\
& \quad+C_{n} \cdot \bar{a}^{3}+D_{n} \cdot \bar{a}^{4}+E_{n} \cdot \bar{a}^{5}+F_{n} \cdot \bar{a}^{6} .
\end{aligned}
$$

As the perturbative expansion is an asymptotic (divergent) series [22], the terms are expected to become smaller up to a certain order, and then become larger again. It is of interest

- at which expansion order this happens,

- how the measured coupling depends on the maximum expansion order,

- how the leading power correction $v_{n} / Q^{l_{n}}$ depends on the maximum expansion order,

- how the minimum perturbative term relates to the power correction.

The best approximation of the theory by an asymptotic series is expected when truncating it near the minimal termincluding additional terms does not necessarily result in a better approximation. Therefore we study the analysis as a function of the truncation order. 


\section{Tests of non-perturbative models}

Our tests use event shape moments with statistical and experimental uncertainties measured by JADE and published in [5] and analogous OPAL data published in [6], covering in total the energy range of 14 to $209 \mathrm{GeV}$. In the JADE data $\mathrm{e}^{+} \mathrm{e}^{-} \rightarrow \mathrm{b} \overline{\mathrm{b}}$ events have been subtracted on a statistical basis. Table 2 gives an overview of the data used.

JADE and OPAL are similar in construction and many parameters [38]. Consistent measurements can be expected from the simultaneous use. The analysis procedures for both data sets were constructed to be similar. Systematic variations of the JADE and OPAL analyses concern detector event reconstruction, selection cuts, Monte Carlo generators and background.

\subsection{Dispersive model}

Our test uses the first five moments of $1-T, B_{\mathrm{T}}, B_{\mathrm{W}}, C$ and $y_{23}^{\mathrm{D}}$, and the second and fourth moment of $M_{\mathrm{H}}$. We compare the prediction with the hadron level data, varying two parameters $\alpha_{\mathrm{S}}\left(M_{\mathrm{Z}^{0}}\right)$ and $\alpha_{0}(2 \mathrm{GeV})$. Figures 1,2 show the comparison of data and predictions (7..11) in the case of $1-T$ and $B_{\mathrm{W}}$. The $\chi^{2} /$ d.o.f. values-calculated

Table 2 Year of data taking, energy range, integrated luminosity, average cms energy and the numbers of selected data events for each JADE [5] or OPAL [6] data sample. The horizontal lines in the OPAL ranges separate the data into the four energy ranges used for fit and presentation purposes

\begin{tabular}{llccr}
\hline Year & $\begin{array}{l}\text { Range of } \\
Q \text { in GeV }\end{array}$ & $\begin{array}{l}\text { Mean } Q \\
\text { in GeV }\end{array}$ & $\begin{array}{l}\text { Luminosity } \\
\left(\mathrm{pb}^{-1}\right)\end{array}$ & $\begin{array}{r}\text { Selected } \\
\text { events }\end{array}$ \\
\hline 1981 & $13.0 \ldots 15.0$ & 14.0 & 1.46 & 1783 \\
1981 & $21.0 \ldots 23.0$ & 22.0 & 2.41 & 1403 \\
1981,1982 & $33.8 \ldots 36.0$ & 34.6 & 61.7 & 14313 \\
1986 & $34.0 \ldots 36.0$ & 35.0 & 92.3 & 20876 \\
1985 & $37.3 \ldots 39.3$ & 38.3 & 8.28 & 1585 \\
1984,1985 & $43.4 \ldots 46.4$ & 43.8 & 28.8 & 4376 \\
\hline \hline 1996,2000 & $91.0 \ldots 91.5$ & 91.3 & 14.7 & 395695 \\
\hline 1995,1997 & $129.9 \ldots 136.3$ & 133.1 & 11.26 & 630 \\
\hline 1996 & $161.2 \ldots 161.6$ & 161.3 & 10.06 & 281 \\
1996 & $170.2 \ldots 172.5$ & 172.1 & 10.38 & 218 \\
1997 & $180.8 \ldots 184.2$ & 182.7 & 57.72 & 1077 \\
\hline 1998 & $188.3 \ldots 189.1$ & 188.6 & 185.2 & 3086 \\
1999 & $191.4 \ldots 192.1$ & 191.6 & 29.53 & 514 \\
1999 & $195.4 \ldots 196.1$ & 195.5 & 76.67 & 1137 \\
1999,2000 & $199.1 \ldots 200.2$ & 199.5 & 79.27 & 1090 \\
1999,2000 & $201.3 \ldots 202.1$ & 201.6 & 37.75 & 519 \\
2000 & $202.5 \ldots 205.5$ & 204.9 & 82.01 & 1130 \\
2000 & $205.5 \ldots 208.9$ & 206.6 & 138.8 & 1717 \\
\hline & & & &
\end{tabular}

from the statistical errors only-vary between 2 and 10, but should be regarded as being indicative only. Experimental differences between JADE and OPAL contribute significantly to the $\chi^{2} /$ d.o.f. values. At low cms energies the predictions drop off again - this unphysical behaviour (not always visible in the figures) substantially contributes to the high $\chi^{2} /$ d.o.f. values. ${ }^{5}$ The moments of the one-hemisphere variables $B_{\mathrm{W}}$ and in particular $M_{\mathrm{H}}$ are described better than in the respective comparison with hadronisation correction by Monte Carlo models [5].

To estimate the experimental systematic uncertainties, the fits are repeated based on the minimum overlap assumption [5] for combining the OPAL and JADE systematic errors. $^{6}$

We study uncertainties of theory parameters by the following variations
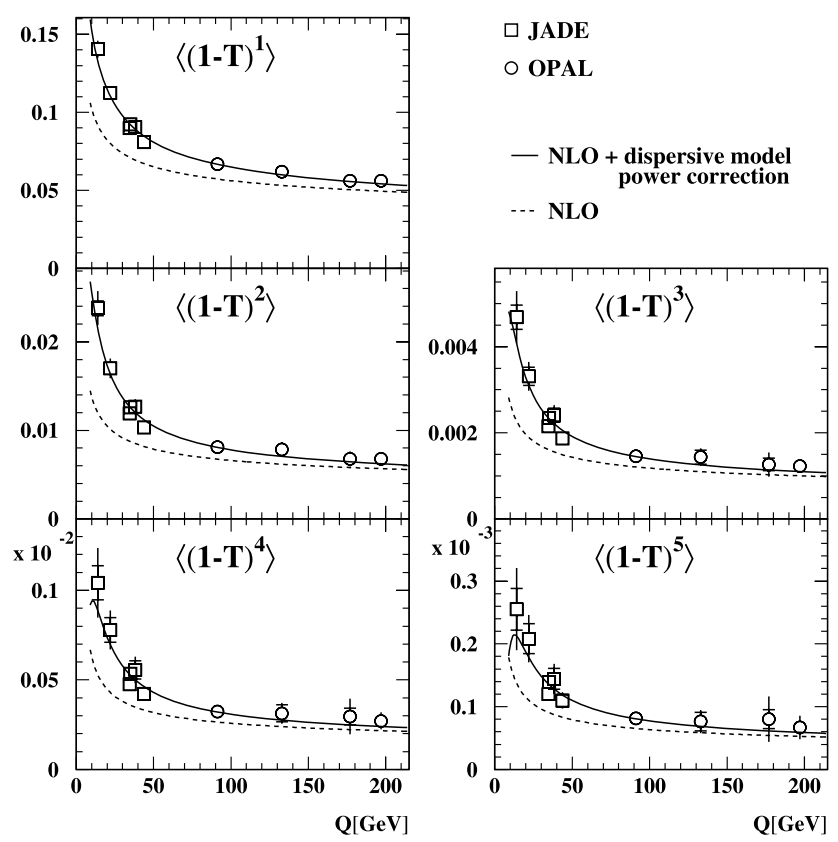

Fig. 1 Fits of the dispersive prediction to JADE and OPAL measurements of $1-T$ moments. The solid line shows the prediction with fitted values of $\alpha_{\mathrm{S}}\left(M_{\mathrm{Z}^{0}}\right)$ and $\alpha_{0}(2 \mathrm{GeV})$, the dashed line shows the pure NLO contribution. The inner error bars show the statistical uncertainties used in the fit, and the outer error bars show the combined statistical and experimental systematic errors

\footnotetext{
${ }^{5}$ The case of $y_{23}^{\mathrm{D}}$ where we compared with the purely perturbative NLO prediction is different. The energy evolution of the data appears consistent with the prediction but the measured higher moments at $14 \mathrm{GeV}$ are too low. A suitable power correction would scale with a higher power of cms energy, and indeed power terms $\ln Q / Q^{2}$ and $1 / Q^{2}$ are expected [7] and describe $y_{23}^{\mathrm{D}}$ distributions better [39].

${ }^{6}$ The $\chi^{2} /$ d.o.f. values from these fits are substantially lower than those from the fits employing the statistical errors.
} 

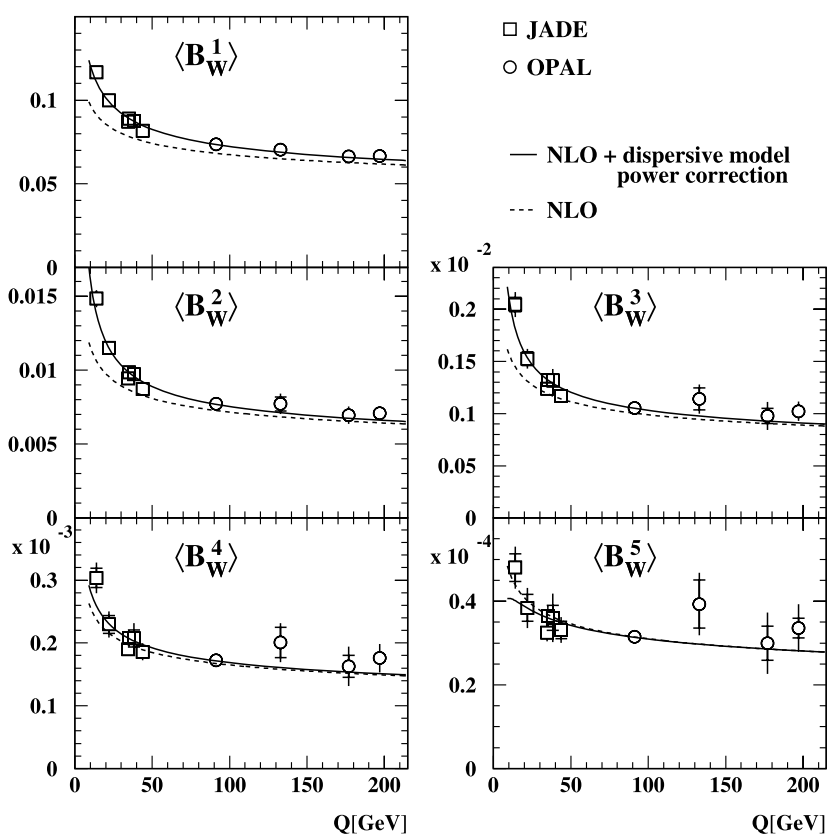

Fig. 2 Fits of the dispersive prediction to JADE and OPAL measurements of $B_{\mathrm{W}}$ moments. The solid line shows the prediction with fitted values of $\alpha_{\mathrm{S}}\left(M_{\mathrm{Z}^{0}}\right)$ and $\alpha_{0}(2 \mathrm{GeV})$, the dashed line shows the pure NLO contribution. The inner error bars show the statistical uncertainties used in the fit, and the outer error bars show the combined statistical and experimental systematic errors

- The renormalisation scale uncertainty (i.e. the effect of higher perturbative orders) is estimated by setting $x_{\mu}=$ 0.5 and $x_{\mu}=2.0$. Our default is always $x_{\mu}=1$.

- The hadronisation uncertainty is estimated by setting

$-\mathcal{M}=1.49 \pm 20 \%$,

$-\mu_{I}=1 \mathrm{GeV}$ and $\mu_{I}=3 \mathrm{GeV}$. The resulting deviations are included into the $\alpha_{\mathrm{S}}\left(M_{\mathrm{Z}^{0}}\right)$ uncertainty only, as $\alpha_{0}\left(\mu_{I}\right)$ depends on $\mu_{I}$ directly by its definition (3).

Tables 3 and 4 and Fig. 3 contain the $\alpha_{\mathrm{S}}\left(M_{\mathrm{Z}^{0}}\right)$ and $\alpha_{0}(2 \mathrm{GeV})$ results from the standard measurement of the moments of the six event shape variables, ${ }^{7}$ and from the $x_{\mu}, \mu_{I}$ and $\mathcal{M}$ variations. The values of $\alpha_{\mathrm{S}}\left(M_{\mathrm{Z}^{0}}\right)$ and $\alpha_{0}(2 \mathrm{GeV})$ measured from $\left\langle(1-T)^{1}\right\rangle,\left\langle C^{1}\right\rangle,\left\langle B_{\mathrm{T}}^{1}\right\rangle,\left\langle\left(y_{23}^{\mathrm{D}}\right)^{1}\right\rangle$, $\left\langle M_{\mathrm{H}}^{2}\right\rangle$ are in agreement within errors with previous analyses, see $[32,40]$ and references therein. The $\alpha_{S}\left(M_{Z^{0}}\right)$ value from $\left\langle B_{\mathrm{W}}^{1}\right\rangle$ is lower and the $\alpha_{0}(2 \mathrm{GeV})$ value higher than in previous analyses because of the incomplete description of $\left\langle B_{\mathrm{W}}^{1}\right\rangle$ in NLO discussed below. This incompleteness affects the predictions at low centre-of-mass energies, leading to high $\alpha_{0}$ values and low $\alpha_{\mathrm{S}}$ values. Low energy data have not yet been analysed with a statistics comparable to ours.

The $\alpha_{\mathrm{S}}\left(M_{\mathrm{Z}^{0}}\right)$ results are similar to those in the analysis employing Monte Carlo models for hadronisation correction $[5,6]$. In particular the $\alpha_{\mathrm{S}}\left(M_{\mathrm{Z}^{0}}\right)$ values steeply rising

\footnotetext{
${ }^{7}$ We list only results from moments that do not show problems in the multi jet region discussed below.
}

with moment order for the variables $1-T, C$ and $B_{\mathrm{T}}$ show the incomplete description of the multi jet region in NLO as already seen and discussed in $[5,6]$. This is also seen in the large renormalisation scale uncertainties. The $\alpha_{0}(2 \mathrm{GeV})$ and $\alpha_{\mathrm{S}}\left(M_{\mathrm{Z}^{0}}\right)$ values from the moments $\left\langle B_{\mathrm{W}}^{n}\right\rangle$ are not universal but decrease with moment order $n$. We can validate only the explicitely calculated $\left\langle B_{\mathrm{W}}^{1}\right\rangle$ prediction.

Based on the statistical errors, the values of $\alpha_{0}(2 \mathrm{GeV})$ from the $\left\langle B_{\mathrm{W}}^{1}\right\rangle,\left\langle M_{\mathrm{H}}^{2}\right\rangle$ and $\left\langle M_{\mathrm{H}}^{4}\right\rangle$ fits are significantly higher than those from $\left\langle(1-T)^{1}\right\rangle,\left\langle C^{1}\right\rangle$ and $\left\langle B_{\mathrm{T}}^{1}\right\rangle$. The NLO description of $B_{\mathrm{W}}$ and $M_{\mathrm{H}}$ in the two jet region is rather incomplete compared to $1-T, C$ and $B_{\mathrm{T}}$ - especially at low energy where the value of the coupling is high. At low $Q$ the NLO predictions of the $B_{\mathrm{W}}, y_{23}^{\mathrm{D}}$ and $M_{\mathrm{H}}$ distributions are (unphysically) negative in a large range of the two jet region $[23,39]$. This incompleteness of the NLO prediction for the moments-in the case of $\left\langle B_{\mathrm{W}}^{1}\right\rangle$ the $\alpha_{\mathrm{S}}^{2}$ coefficient is even negative-is compensated by a larger power correction. The perturbative description then contributes less as is seen by the low $\alpha_{\mathrm{S}}\left(M_{\mathrm{Z}^{0}}\right)$ values. Fits to JADE data of $\left\langle B_{\mathrm{W}}^{1}\right\rangle$ alone give $\alpha_{\mathrm{S}}\left(M_{\mathrm{Z}^{0}}\right)=0.1029 \pm 0.0016$, $\alpha_{0}(2 \mathrm{GeV})=0.728 \pm 0.017$; to OPAL alone they return $\alpha_{\mathrm{S}}\left(M_{\mathrm{Z}^{0}}\right)=0.1242 \pm 0.0025, \alpha_{0}(2 \mathrm{GeV})=0.234 \pm 0.103$ (statistical errors). So at low energies where the coupling is large, the compensation is stronger, and the power correction is not universal (JADE and OPAL agree better on every other moment).

For averaging $\alpha_{\mathrm{S}}\left(M_{\mathrm{Z}^{0}}\right)$ and $\alpha_{0}(2 \mathrm{GeV})$ over many variables and moment orders, we exclude moments that suffer from the problems discussed in the preceding two paragraphs: The deficiencies in the description of the multi jet region for the two-hemisphere variables have already been seen in [5, 6]. To select observables with an apparently converging perturbative prediction, we consider as in $[5,6]$ only those results for which the NLO term in (2) is less than half the corresponding LO term (i.e. $|K|<25$ or $\left.\left|K \alpha_{\mathrm{S}} / 2 \pi\right|<0.5\right)$, with $K=\mathcal{B}_{n} / \mathcal{A}_{n}$. The $K$ values are shown in Refs. [5, 6].

The higher moments of the jet broadenings are excluded because of the incomplete [30] description of their distributions in general. As in [5] the first moment of $B_{\mathrm{W}}$ is excluded because the universality of the fit parameters could not be confirmed.

Thus we combine the results of $\alpha_{\mathrm{S}}\left(M_{\mathrm{Z}^{0}}\right)$ and $\alpha_{0}(2 \mathrm{GeV})$ from the moments $\left\langle(1-T)^{1}\right\rangle,\left\langle C^{1}\right\rangle,\left\langle B_{\mathrm{T}}^{1}\right\rangle$, $\left\langle\left(y_{23}^{\mathrm{D}}\right)^{1}\right\rangle \ldots\left\langle\left(y_{23}^{\mathrm{D}}\right)^{5}\right\rangle$, and $\left\langle M_{\mathrm{H}}^{2}\right\rangle,\left\langle M_{\mathrm{H}}^{4}\right\rangle$. The parameters are consistent within total errors, and the combination procedure follows that used in $[5,6] .{ }^{8}$ The correlations

\footnotetext{
${ }^{8}$ The covariances of the statistical errors are estimated by PYTHIA at hadron level at $91.2 \mathrm{GeV}$, the covariances of the experimental systematic uncertainties are taken as $E_{i j}=\operatorname{Min}\left\{\sigma_{\text {exp. }, i}^{2}, \sigma_{\text {exp., }, j}^{2}\right\}$, and hadronisation and renormalisation scale uncertainties are found by repeating the analysis with varied parameters.
} 
Table 3 Measurements of $\alpha_{\mathrm{S}}\left(M_{\mathrm{Z}^{0}}\right)$ and $\alpha_{0}(2 \mathrm{GeV})$ in the dispersive model from first moments of six event shape variables over the full analysed range of cms energy, $14 \ldots 209 \mathrm{GeV}$. The $\chi^{2} /$ d.o.f. values are based on the statistical errors only, see text for further discussion

\begin{tabular}{|c|c|c|c|c|c|}
\hline & $\left\langle(1-T)^{1}\right\rangle$ & $\left\langle C^{1}\right\rangle$ & $\left\langle B_{\mathrm{T}}^{1}\right\rangle$ & $\left\langle B_{\mathrm{W}}^{1}\right\rangle$ & $\left\langle\left(y_{23}^{\mathrm{D}}\right)^{1}\right\rangle$ \\
\hline$\alpha_{\mathrm{S}}\left(M_{\mathrm{Z}^{0}}\right)$ & 0.1234 & 0.1228 & 0.1200 & 0.1142 & 0.1181 \\
\hline Statistical error & 0.0006 & 0.0004 & 0.0005 & 0.0006 & 0.0004 \\
\hline Experimental syst. & 0.0015 & 0.0012 & 0.0011 & 0.0015 & 0.0016 \\
\hline$x_{\mu}$ variation: $x_{\mu}=2.0$ & +0.0073 & +0.0073 & +0.0047 & +0.0004 & +0.0050 \\
\hline$x_{\mu}=0.5$ & -0.0062 & -0.0063 & -0.0041 & +0.0009 & -0.0038 \\
\hline$\mu_{I}$ variation: $\mu_{I}=1 \mathrm{GeV}$ & +0.0025 & +0.0030 & +0.0017 & +0.0008 & - \\
\hline$\mu_{I}=3 \mathrm{GeV}$ & -0.0019 & -0.0022 & -0.0013 & -0.0007 & - \\
\hline $\mathcal{M}$ variation: $\mathcal{M}-20 \%$ & +0.0011 & +0.0014 & +0.0008 & +0.0004 & - \\
\hline $\mathcal{M}+20 \%$ & -0.0011 & -0.0013 & -0.0007 & -0.0004 & - \\
\hline Theoretical syst. & 0.0078 & 0.0080 & 0.0051 & 0.0012 & 0.0050 \\
\hline$\alpha_{0}(2 \mathrm{GeV})$ & 0.490 & 0.433 & 0.514 & 0.604 & - \\
\hline Statistical error & 0.007 & 0.003 & 0.009 & 0.015 & - \\
\hline Experimental syst. & 0.010 & 0.005 & 0.009 & 0.014 & - \\
\hline$x_{\mu}$ variation: $x_{\mu}=2.0$ & -0.022 & -0.024 & +0.013 & -0.049 & - \\
\hline$x_{\mu}=0.5$ & +0.031 & +0.033 & +0.011 & +0.147 & - \\
\hline $\mathcal{M}$ variation: $\mathcal{M}-20 \%$ & +0.047 & +0.034 & +0.053 & +0.086 & - \\
\hline $\mathcal{M}+20 \%$ & -0.033 & -0.025 & -0.037 & -0.057 & - \\
\hline Theoretical syst. & 0.056 & 0.048 & 0.055 & 0.170 & - \\
\hline Correlation $\alpha_{\mathrm{S}}\left(M_{\mathrm{Z}^{0}}\right), \alpha_{0}(2 \mathrm{GeV})$ & -0.86 & -0.80 & -0.93 & -0.90 & - \\
\hline$\chi^{2} /$ d.o.f. & $47.8 / 10$ & $66.3 / 10$ & $106 / 10$ & $75.3 / 10$ & $37.7 / 10$ \\
\hline
\end{tabular}

Table 4 Measurements of $\alpha_{\mathrm{S}}\left(M_{\mathrm{Z}^{0}}\right)$ and $\alpha_{0}(2 \mathrm{GeV})$ in the dispersive model from second to fifth moments of six event shape variables over the full analysed range of cms energy, $14 \ldots 209 \mathrm{GeV}$. The $\chi^{2} /$ d.o.f. values are based on the statistical errors only, see text for further discussion

\begin{tabular}{|c|c|c|c|c|c|c|}
\hline & $\left\langle\left(y_{23}^{\mathrm{D}}\right)^{2}\right\rangle$ & $\left\langle M_{\mathrm{H}}^{2}\right\rangle$ & $\left\langle\left(y_{23}^{\mathrm{D}}\right)^{3}\right\rangle$ & $\left\langle\left(y_{23}^{\mathrm{D}}\right)^{4}\right\rangle$ & $\left\langle M_{\mathrm{H}}^{4}\right\rangle$ & $\left\langle\left(y_{23}^{\mathrm{D}}\right)^{5}\right\rangle$ \\
\hline$\alpha_{\mathrm{S}}\left(M_{Z^{0}}\right)$ & 0.1182 & 0.1158 & 0.1173 & 0.1160 & 0.1154 & 0.1144 \\
\hline Statistical error & 0.0009 & 0.0007 & 0.0013 & 0.0017 & 0.0010 & 0.0020 \\
\hline Experimental syst. & 0.0021 & 0.0024 & 0.0020 & 0.0025 & 0.0034 & 0.0033 \\
\hline$x_{\mu}$ variation: $x_{\mu}=2.0$ & +0.0054 & +0.0042 & +0.0053 & +0.0050 & +0.0051 & +0.0047 \\
\hline$x_{\mu}=0.5$ & -0.0042 & -0.0033 & -0.0041 & -0.0038 & -0.0042 & -0.0036 \\
\hline$\mu_{I}$ variation: $\mu_{I}=1 \mathrm{GeV}$ & - & +0.0013 & - & - & +0.0011 & - \\
\hline$\mu_{I}=3 \mathrm{GeV}$ & - & -0.0010 & - & - & -0.0009 & - \\
\hline $\mathcal{M}$ variation: $\mathcal{M}-20 \%$ & - & +0.0006 & - & - & +0.0005 & - \\
\hline $\mathcal{M}+20 \%$ & - & -0.0006 & - & - & -0.0005 & - \\
\hline Theoretical syst. & 0.0054 & 0.0045 & 0.0053 & 0.0050 & 0.0053 & 0.0047 \\
\hline$\alpha_{0}(2 \mathrm{GeV})$ & - & 0.601 & - & - & 0.579 & - \\
\hline Statistical error & - & 0.011 & - & - & 0.015 & - \\
\hline Experimental syst. & - & 0.012 & - & - & 0.022 & - \\
\hline$x_{\mu}$ variation: $x_{\mu}=2.0$ & - & -0.022 & - & - & -0.022 & - \\
\hline$x_{\mu}=0.5$ & - & +0.037 & - & - & +0.034 & - \\
\hline $\mathcal{M}$ variation: $\mathcal{M}-20 \%$ & - & +0.083 & - & - & +0.079 & - \\
\hline $\mathcal{M}+20 \%$ & - & -0.056 & - & - & -0.054 & - \\
\hline Theoretical syst. & - & 0.091 & - & - & 0.086 & - \\
\hline Correlation $\alpha_{\mathrm{S}}\left(M_{\mathrm{Z}^{0}}\right), \alpha_{0}(2 \mathrm{GeV})$ & - & -0.88 & - & - & -0.82 & - \\
\hline$\chi^{2} /$ d.o.f. & $18.3 / 10$ & $25.2 / 10$ & $21.0 / 10$ & $18.7 / 10$ & $26.4 / 10$ & $15.2 / 10$ \\
\hline
\end{tabular}


between $\alpha_{\mathrm{S}}\left(M_{\mathrm{Z}^{0}}\right)$ and $\alpha_{0}(2 \mathrm{GeV})$ have rather constant values from -0.80 to -0.93 and thus the combination is done individually for $\alpha_{\mathrm{S}}\left(M_{\mathrm{Z}^{0}}\right)$ and $\alpha_{0}(2 \mathrm{GeV})$. The averages are

$$
\begin{aligned}
\alpha_{\mathrm{S}}\left(M_{\mathrm{Z}^{0}}\right)= & 0.1183 \pm 0.0007 \text { (stat. }) \pm 0.0016(\text { exp. }) \\
& \pm 0.0011(\text { had. })_{-0.0042}^{+0.0052}\left(x_{\mu}\right) \\
= & 0.1183 \pm 0.0056 \text { (tot.) } \\
\alpha_{0}(2 \mathrm{GeV})= & 0.493 \pm 0.006 \text { (stat.) } \pm 0.008(\text { exp. }) \\
& \pm 0.050 \text { (had. })_{-0.014}^{+0.028}\left(x_{\mu}\right) \\
= & 0.493 \pm 0.058 \text { (tot.). }
\end{aligned}
$$

Mass effects, in particular on $M_{\mathrm{H}}$ moments, have not been considered in obtaining the averages for $\alpha_{\mathrm{S}}$ and $\alpha_{0}$. However, [41] shows that mass effects are smaller than effects due to the non-inclusiveness of the heavy jet mass, used in this analysis. Moreover, the LEP analysis [42] found mass effects affecting $\alpha_{\mathrm{S}}$ at the level of the statistical error while those effects are in the order of $30 \%$ for $\alpha_{0}$.

\subsection{Shape function}

In [8] values for the shape function parameters were determined by comparing a distribution of $M_{\mathrm{H}}^{2}$ at $91 \mathrm{GeV}$ with the NLO + NLLA prediction combined with power terms from the shape function to be $\lambda_{1}=1.22 \mathrm{GeV}, \lambda_{2}=$

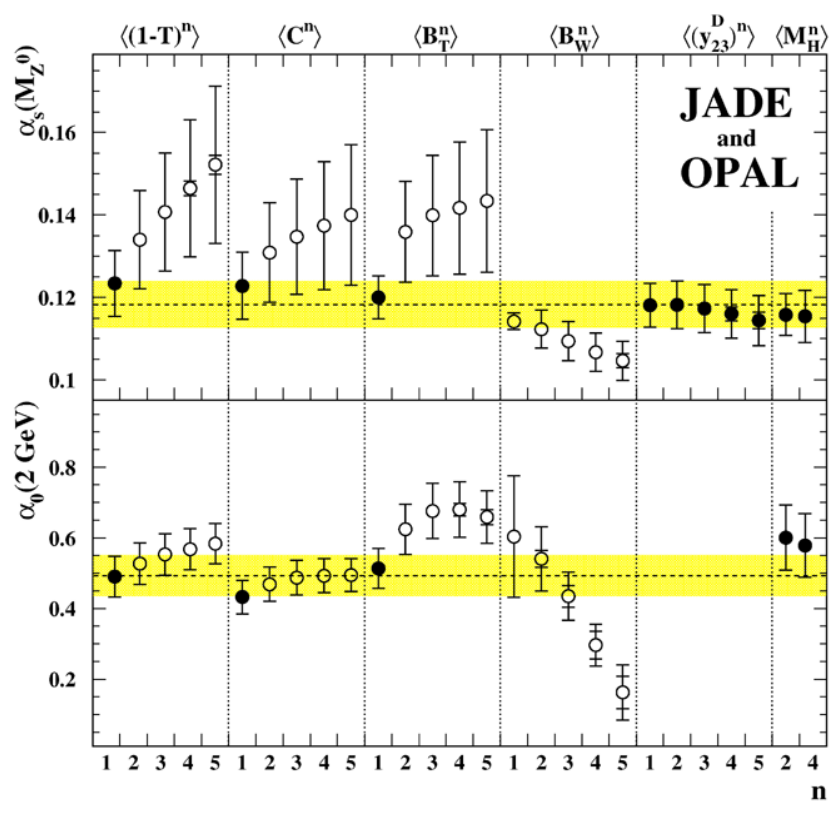

Fig. 3 Measurements of $\alpha_{\mathrm{S}}\left(M_{\mathrm{Z}^{0}}\right)$ and $\alpha_{0}(2 \mathrm{GeV})$ from moments of six event shape variables at PETRA and LEP energies. The inner error bars-where visible - show the statistical errors, the outer bars show the total errors. The dashed lines indicate the weighted averages, the shaded bands show their errors. Only the measurements indicated by solid symbols are used for the averages
$1.70 \mathrm{GeV}^{2}$ and $\delta \lambda_{2}$ from $\delta \lambda_{2}(10 \mathrm{GeV})=1.4(\mathrm{GeV})^{2}$ to $\delta \lambda_{2}(100 \mathrm{GeV})=1.2(\mathrm{GeV})^{2}$.

We perform fits to the moments $\left\langle(1-T)^{1}\right\rangle,\left\langle C^{1}\right\rangle$ and $\left\langle M_{\mathrm{H}}^{2}\right\rangle$ with two free parameters $\alpha_{\mathrm{S}}\left(M_{\mathrm{Z}^{0}}\right)$ and $\lambda_{1}$. Fits to the higher moments $\left\langle(1-T)^{2}\right\rangle,\left\langle C^{2}\right\rangle$ and $\left\langle M_{\mathrm{H}}^{4}\right\rangle$ are done with an additional parameter. Because of the weak energy dependence of $\delta \lambda_{2}$ in (22) we substitute the numerator $\lambda_{2}+\delta \lambda_{2}(Q)$ by the fit parameter $\bar{\lambda}_{2}$. The fits to $\left\langle C^{2}\right\rangle$ and $\left\langle M_{\mathrm{H}}^{4}\right\rangle$ are not sensitive to the parameter $\bar{\lambda}_{2}$ while the fit to $\left\langle(1-T)^{2}\right\rangle$ gives $\bar{\lambda}_{2}=(1.2 \pm 1.3)(\mathrm{GeV})^{2}$. For further analysis we therefore set $\bar{\lambda}_{2}=0$.

Figure 4 shows the comparison of the data with the prediction. The data are fitted well with $\chi^{2} /$ d.o.f.-based on the statistical errors-in the order of one. As before, the experimental systematic uncertainties are estimated by the minimum overlap assumption, and the renormalisation scale uncertainty by setting $x_{\mu}=0.5$ and $x_{\mu}=2.0$. Table 5 and
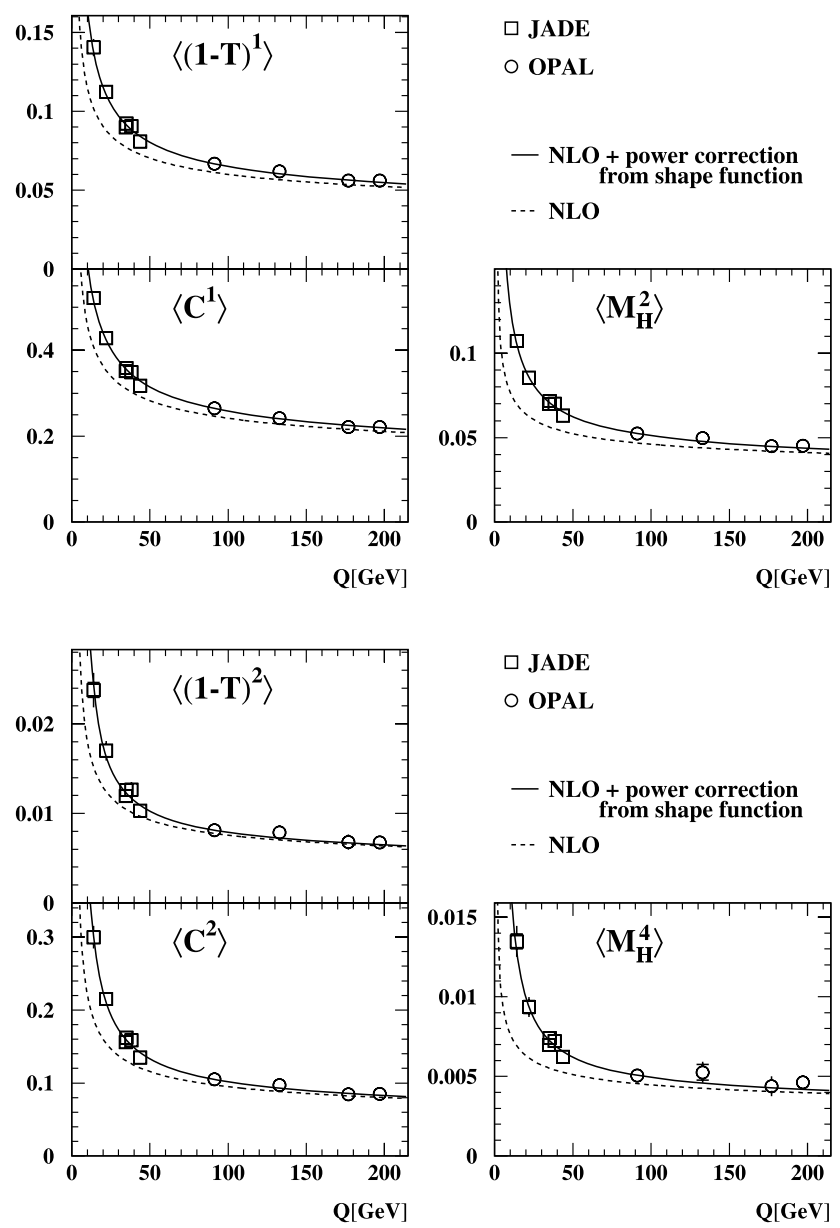

Fig. 4 Fits of the shape function prediction with $\bar{\lambda}_{2}=0$ to JADE and OPAL measurements of first and second moments of $1-T$ and $C$ and second and fourth moments of $M_{\mathrm{H}}$. The solid line shows the fitted prediction and the dashed line shows the pure NLO contribution. The inner error bars show the statistical uncertainties used in the fit and the outer error bars show the combined statistical and experimental systematic errors. Most of the error bars are smaller than the data points 
Table 5 Measurements of $\alpha_{\mathrm{S}}\left(M_{\mathrm{Z}^{0}}\right)$ and $\lambda_{1}$ in the shape function model from the first and second moments of three event shape variables (second and fourth moment in case of $M_{\mathrm{H}}$ ) over the full analysed range of cms energy, 14 ...209 GeV

\begin{tabular}{|c|c|c|c|}
\hline & $\left\langle(1-T)^{1}\right\rangle$ & $\left\langle C^{1}\right\rangle$ & $\left\langle M_{\mathrm{H}}^{2}\right\rangle$ \\
\hline$\alpha_{\mathrm{S}}\left(M_{Z^{0}}\right)$ & 0.1304 & 0.1305 & 0.1193 \\
\hline Statistical error & 0.0008 & 0.0006 & 0.0008 \\
\hline Experimental syst. & 0.0018 & 0.0015 & 0.0027 \\
\hline$x_{\mu}$ variation: $x_{\mu}=2.0$ & +0.0069 & +0.0064 & +0.0038 \\
\hline$x_{\mu}=0.5$ & -0.0054 & -0.0050 & -0.0025 \\
\hline$\lambda_{1}[\mathrm{GeV}]$ & 0.499 & 0.393 & 1.010 \\
\hline Statistical error & 0.034 & 0.024 & 0.047 \\
\hline Experimental syst. & 0.065 & 0.045 & 0.075 \\
\hline$x_{\mu}$ variation: $x_{\mu}=2.0$ & +0.053 & +0.063 & +0.044 \\
\hline$x_{\mu}=0.5$ & -0.067 & -0.075 & -0.045 \\
\hline Correlation $\alpha_{\mathrm{S}}\left(M_{\mathrm{Z}^{0}}\right), \lambda_{1}$ & -0.96 & -0.96 & -0.94 \\
\hline \multirow[t]{2}{*}{$\chi^{2} /$ d.o.f. } & $23.3 / 8$ & $22.6 / 8$ & $19.6 / 8$ \\
\hline & $\left\langle(1-T)^{1}\right\rangle$ & $\left\langle C^{1}\right\rangle$ & $\left\langle M_{\mathrm{H}}^{2}\right\rangle$ \\
\hline$\alpha_{\mathrm{S}}\left(M_{Z^{0}}\right)$ & 0.1426 & 0.1456 & 0.1184 \\
\hline Statistical error & 0.0014 & 0.0008 & 0.0012 \\
\hline Experimental syst. & 0.0028 & 0.0023 & 0.0041 \\
\hline$x_{\mu}$ variation: $x_{\mu}=2.0$ & +0.0111 & +0.0117 & +0.0049 \\
\hline$x_{\mu}=0.5$ & -0.0091 & -0.0097 & -0.0037 \\
\hline$\lambda_{1}[\mathrm{GeV}]$ & 0.516 & 0.364 & 0.964 \\
\hline Statistical error & 0.056 & 0.023 & 0.079 \\
\hline Experimental syst. & 0.096 & 0.052 & 0.133 \\
\hline$x_{\mu}$ variation: $x_{\mu}=2.0$ & +0.044 & +0.063 & +0.031 \\
\hline$x_{\mu}=0.5$ & -0.052 & -0.054 & -0.030 \\
\hline Correlation $\alpha_{\mathrm{S}}\left(M_{\mathrm{Z}^{0}}\right), \lambda_{1}$ & -0.95 & -0.91 & -0.92 \\
\hline$\chi^{2} /$ d.o.f. & $20.9 / 8$ & $18.3 / 8$ & $20.2 / 8$ \\
\hline
\end{tabular}

Fig. 5 contain the results for $\alpha_{\mathrm{S}}\left(M_{\mathrm{Z}^{0}}\right)$ and $\lambda_{1}$ from the standard measurement and the systematic variations.

The values of $\alpha_{S}\left(M_{Z^{0}}\right)$ show the typical increase for the higher moments of $1-T$ and $C$. The values of $\lambda_{1}$ are consistent for the first two moments of every event shape variable. Furthermore they are consistent for the twohemisphere variables $1-T$ and $C$. However, the values from $M_{\mathrm{H}}$ are not compatible. As the respective predictions are similar, we refer to the high values of $\alpha_{0}(2 \mathrm{GeV})$ from the moments $\left\langle M_{\mathrm{H}}^{2}\right\rangle$ and $\left\langle M_{\mathrm{H}}^{4}\right\rangle$ in the dispersive model, see Sect. 4.1. The large power corrections compensate for the incomplete perturbative NLO description. Since the values are not consistent and no systematic evaluation of the theory uncertainty is available, we do not combine any of the fit parameters.

The $\lambda_{1}$ values from $1-T, C$ and, less pronounced, from $M_{\mathrm{H}}$ are smaller than the results in [8]. A substantial difference of [8] with our work is the inclusion of the NLLA approximation in the distribution prediction. The NNLA terms have unphysically negative divergences in the extreme twojet region. The mean values are sensitive to this region, and therefore they are not described reliably by NLLA theory. The NLLA resummation has negligible influence on the higher moments.

\subsection{Variance of event shape variables}

From the first and second moments of event shape variables $y$ the variance $\operatorname{Var}(y)=\left\langle y^{2}\right\rangle-\langle y\rangle^{2}$ has been calculated [43]. The experimental uncertainties have been determined by systematic variations analogously to the moments at hadron level $[5,6]$. The variance of $C, B_{\mathrm{T}}, B_{\mathrm{W}}, M_{\mathrm{H}}$ and $M_{\mathrm{H}}^{2}$ becomes larger with rising $Q$ while the variance of $1-T$ becomes smaller, see ${ }^{9}$ Fig. 6 and [43]. Studying other observables defined in [6] we find that the variance of $T_{\text {maj. }}$ and $O$ becomes larger with rising $Q$ while the variance of $T_{\min }, S, M_{\mathrm{L}}$ and $B_{\mathrm{N}}$ becomes smaller, see [43]. This behaviour is determined by the multi jet region of the distri-

\footnotetext{
${ }^{9}$ In case of $1-T$ this is not seen very clearly in the plot, but significantly in a straight line fit.
} 


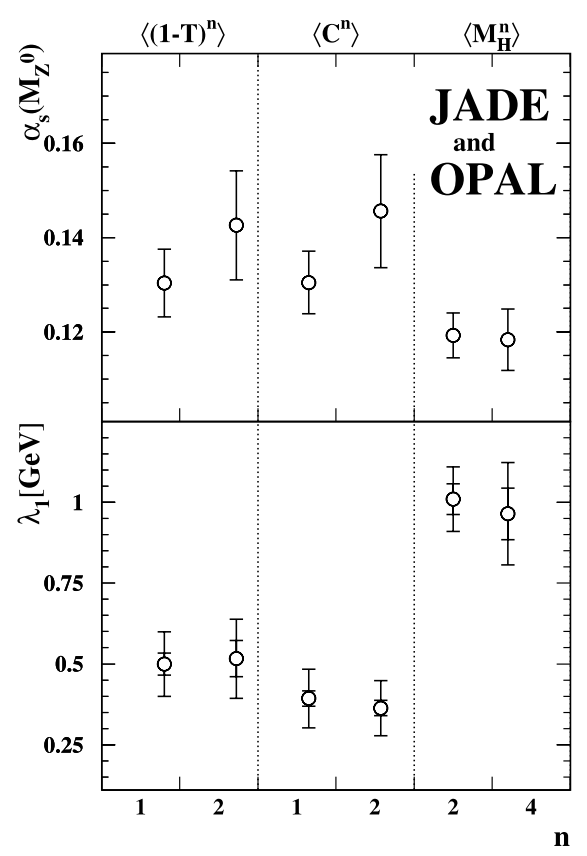

Fig. 5 Measurements of $\alpha_{\mathrm{S}}\left(M_{\mathrm{Z}^{0}}\right)$ and $\lambda_{1}$ from moments of three event shape variables at PETRA and LEP energies. The inner error bars-where visible - show the statistical errors, the outer bars show the total errors

bution [44]. The evolution is reproduced by Monte Carlo models qualitatively well; however data and models differ by typically up to five standard deviations.

Figure 6 shows the comparison of the prediction (23) using the dispersive model and data of $1-T, C, B_{\mathrm{T}}, B_{\mathrm{W}}, y_{23}^{\mathrm{D}}$ and $M_{\mathrm{H}}^{2}$. The fits of the free parameter $\alpha_{\mathrm{S}}\left(M_{\mathrm{Z}^{0}}\right)$ use central values of the measurements and the statistical errors. The energy evolution of prediction and data for $\operatorname{Var}\left(B_{\mathrm{W}}\right)$, $\operatorname{Var}(1-T)$, and $\operatorname{Var}\left(y_{23}^{\mathrm{D}}\right)$ coincides qualitatively (in case of $\operatorname{Var}\left(y_{23}^{\mathrm{D}}\right)$ except for the lowest energy points of 14 and $22 \mathrm{GeV})$, but not for $\operatorname{Var}(C), \operatorname{Var}\left(B_{\mathrm{T}}\right)$ and $\operatorname{Var}\left(M_{\mathrm{H}}^{2}\right)$. The $\chi^{2} /$ d.o.f. values are large and the $\alpha_{\mathrm{S}}\left(M_{\mathrm{Z}^{0}}\right)$ values are not compatible with each other or with established QCD analyses.

The predictions using the shape function differ only for $\operatorname{Var}(C)$ from the dispersive model. Figure 7 shows the comparison of this prediction (24) and data. The fit gives $\chi^{2} /$ d.o.f. $=50 / 8, \alpha_{\mathrm{S}}\left(M_{\mathrm{Z}^{0}}\right)=0.0963 \pm 0.0004$ and $\lambda_{1}=$ $(0.824 \pm 0.025) \mathrm{GeV}$ (statistical errors). The correlation of $\alpha_{\mathrm{S}}\left(M_{\mathrm{Z}^{0}}\right)$ and $\lambda_{1}$ is 0.80 . In the shape function description the $Q$ evolution of the perturbative description deviating from the data is corrected at low energies by the term $\propto \lambda_{1}$. Thus the steep energy evolution at low $Q$ is reproduced successfully. However, the fitted values $\alpha_{\mathrm{S}}\left(M_{\mathrm{Z}^{0}}\right)$ and $\lambda_{1}$ are not compatible with the corresponding values from $\left\langle C^{1}\right\rangle$ or $\left\langle C^{2}\right\rangle$.
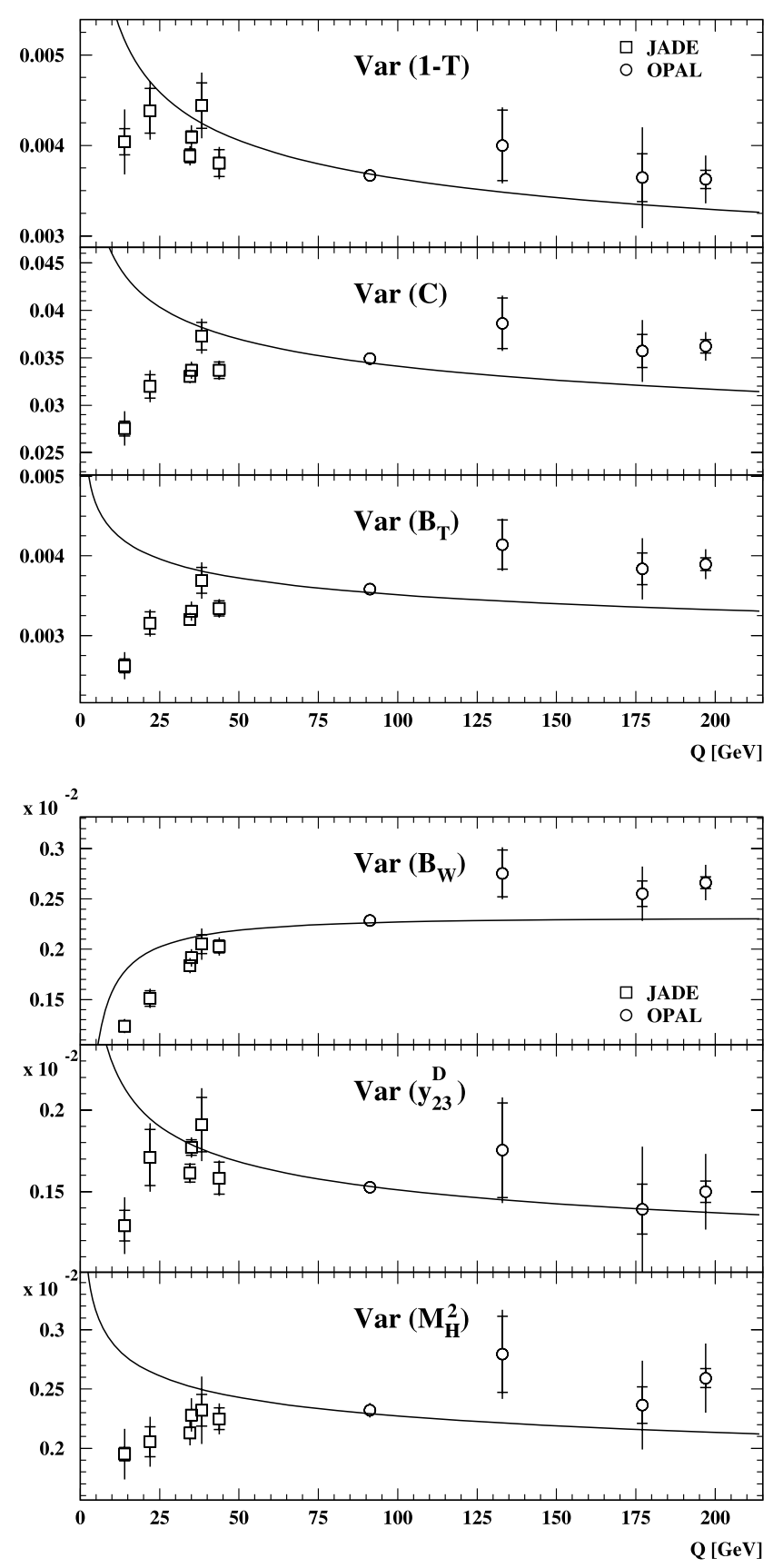

Fig. 6 Comparison of the NLO prediction with JADE and OPAL measurements of the variance of $1-T, C, B_{\mathrm{T}}, B_{\mathrm{W}}, y_{23}^{\mathrm{D}}$ and $M_{\mathrm{H}}^{2}$. The inner error bars show the statistical uncertainties used in the fit and the outer error bars show the combined statistical and experimental systematic errors. The line shows the NLO prediction with fitted value of $\alpha_{\mathrm{S}}\left(M_{\mathrm{Z}^{0}}\right)$

\subsection{Single dressed gluon approximation}

The hadron level data are compared with the five different predictions which result from truncating (28) after or$\operatorname{der} \mathcal{O}\left(\bar{a}^{2}\right) \ldots \mathcal{O}\left(\bar{a}^{6}\right)$. The data are described by the predic- 


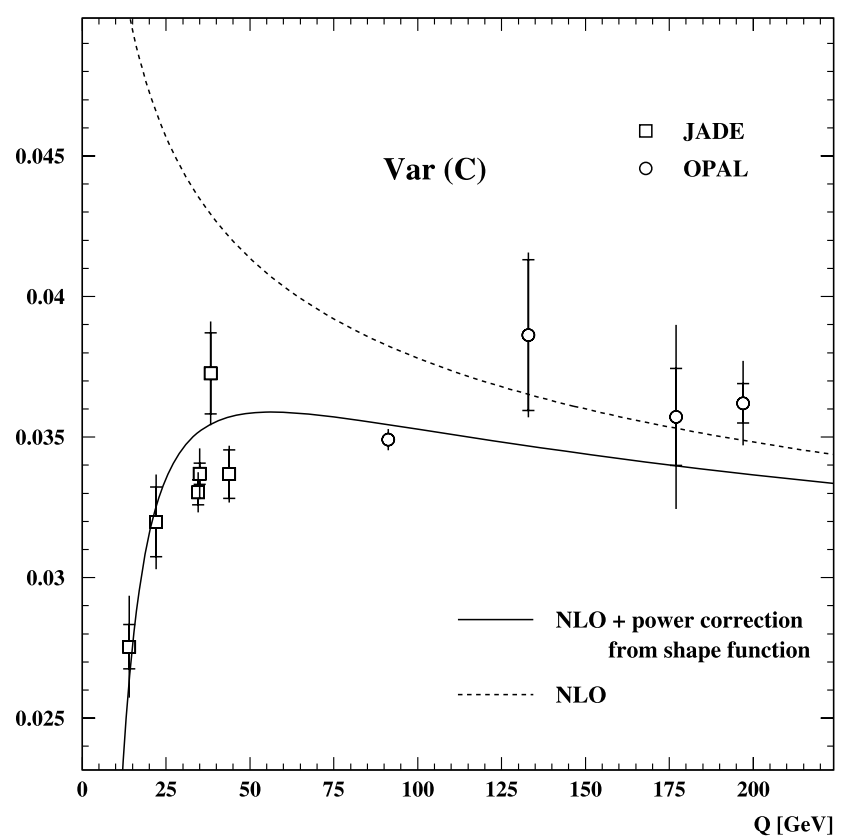

Fig. 7 Comparison of the shape function prediction with JADE and OPAL measurements of the variance of $C$. The inner error bars show the statistical uncertainties used in the fit and the outer error bars show the combined statistical and experimental systematic errors. The solid line shows the prediction with fitted values of $\alpha_{\mathrm{S}}\left(M_{\mathrm{Z}^{0}}\right)$ and $\lambda_{1}$, the dashed line shows the pure NLO contribution

tions well. Figure 8 shows the comparison ${ }^{10}$ of data and prediction for truncating after $\bar{a}^{5}$. The fit results for $\alpha_{\mathrm{S}}$ and $v_{i}$ are given in Table 6 . The coefficients $\kappa_{2} \ldots \kappa_{4}$ of the more strongly suppressed power correction in all instances turn out to be compatible with zero.

For every moment order we study specific properties to find the series describing the data best. The $\chi^{2} /$ d.o.f. values from the various expansion orders do not differ significantly for any moment order and thus do not discriminate between different expansion orders.

$\left\langle(1-T)^{1}\right\rangle$ : For the first thrust moment at the order $m_{\max }=$ 5 the power correction is compatible with zero, then it becomes negative. ${ }^{11}$ The correction added to $\alpha_{\mathrm{S}}$ when going from the fourth to the fifth expansion order is minimal, the values of $\alpha_{\mathrm{S}}\left(M_{\mathrm{Z}^{0}}\right)$ and the power correction reach their minimum at $m_{\max }=5$. So the convergence appears best at $m_{\max }=5$.

$\left\langle(1-T)^{2}\right\rangle$ : Including higher perturbative orders the $\alpha_{\mathrm{S}}\left(M_{\mathrm{Z}^{0}}\right)$ and $\nu_{2}$ values from the second thrust moment decrease. The purely perturbative description appears continually more accurate, we therefore choose $m_{\max }=6$.

\footnotetext{
${ }^{10}$ Comparisons for second to sixth expansion order, and more discussion, can be found in [44].

${ }^{11}$ There is no distinct sign of the power term. It can be positive or negative, or change its sign when using different regularisations [35].
}

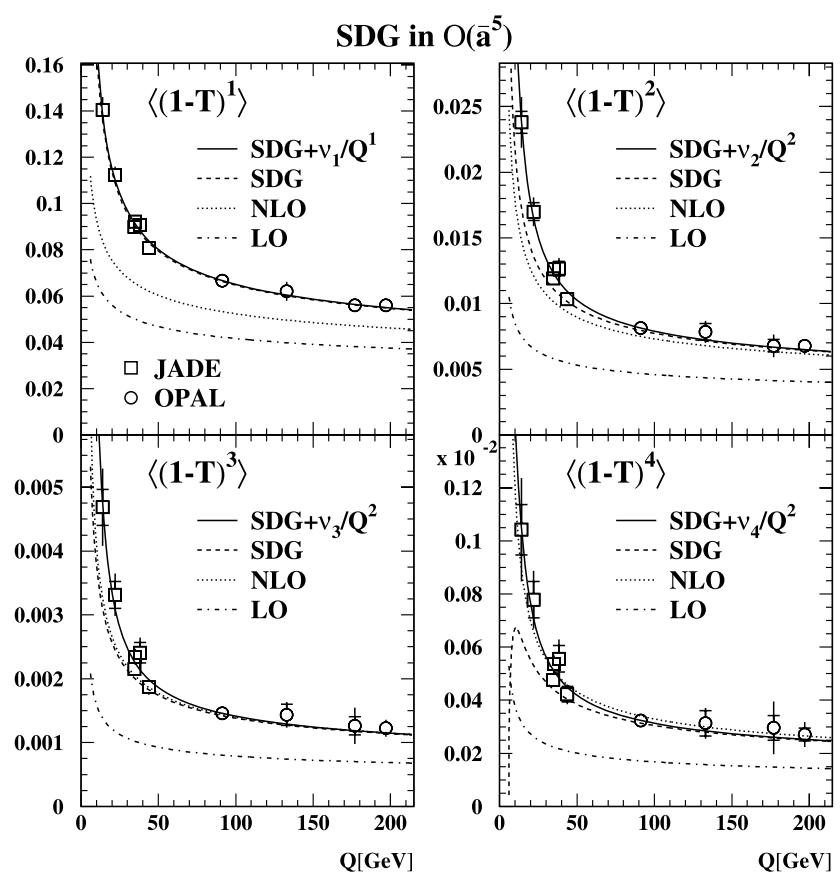

Fig. 8 Fits of the SDG prediction including power correction to JADE and OPAL measurements of first to fourth moment of $1-T$. The perturbative part of the prediction has been calculated in $\mathcal{O}\left(\bar{a}^{5}\right)$. Superimposed are the perturbative prediction in leading, next-to-leading, and maximum order as indicated in the figure. The inner error bars show the statistical uncertainties used in the fit, the outer error bars show the combined statistical and experimental systematic errors

$\left\langle(1-T)^{3}\right\rangle,\left\langle(1-T)^{4}\right\rangle$ : Fitting the third or fourth thrust moment, to compensate the negative perturbative terms the power correction increases when increasing the truncation order. Thus the NLO description appears more accurate than the SDG approximation, and we choose $m_{\max }=2$.

Figure 9 compares the perturbative terms and the power correction terms. In case of the first moment the power correction is compatible with zero; the purely perturbative prediction from the SDG approximation already describes the data well. The power correction is larger for the third and fourth moments, but is still small compared to the LO and NLO perturbative terms down to $Q=10 \mathrm{GeV}$. In case of the second moment the higher SDG terms up to $\mathcal{O}\left(\bar{a}^{6}\right)$ are comparable with the power correction.

\subsection{Measuring $\alpha_{\mathrm{S}}\left(M_{\mathrm{Z}^{0}}\right)$ in the SDG approximation}

The $\alpha_{\mathrm{S}}\left(M_{\mathrm{Z}^{0}}\right)$ values steeply rise with moment order in this model as observed before in Subsects. 4.1, 4.2. The problem of describing the higher thrust moments consistently-i.e. with compatible fit parameters-is not solved in this model. We measure the strong coupling in this model only from $\left\langle(1-T)^{1}\right\rangle$.

The central value of the measurement and its statistical error are shown in Table 6 as case $m_{\max }=5$. To determine 
Table 6 Measurements of $\alpha_{\mathrm{S}}\left(M_{\mathrm{Z}^{0}}\right)$ and $v_{n}$ from first to fourth moment of $1-T$ over the full analysed range of $\mathrm{cms}$ energy, 14 .. $209 \mathrm{GeV}$. The single dressed gluon approximation is used in maximum order $m_{\max }$. The errors are statistical. Units of $v_{n}$ are $\mathrm{GeV}$ for $n=1$ and $(\mathrm{GeV})^{2}$ for $n=2,3,4$

\begin{tabular}{|c|c|c|c|c|c|}
\hline & $m_{\max }$ & $\alpha_{\mathrm{S}}\left(M_{\mathrm{Z}^{0}}\right)$ & $v_{n}$ & $\begin{array}{l}\text { Correlation } \\
\alpha_{\mathrm{S}}\left(M_{\mathrm{Z}^{0}}\right), v_{n}\end{array}$ & $\chi^{2} /$ d.o.f. \\
\hline \multirow[t]{5}{*}{$\left\langle(1-T)^{1}\right\rangle$} & 2 & $0.1314 \pm 0.0008$ & $0.430 \pm 0.036$ & -0.96 & $29.1 / 10$ \\
\hline & 3 & $0.1209 \pm 0.0007$ & $0.352 \pm 0.039$ & -0.97 & $30.0 / 10$ \\
\hline & 4 & $0.1178 \pm 0.0007$ & $0.223 \pm 0.045$ & -0.97 & $29.3 / 10$ \\
\hline & 5 & $0.1172 \pm 0.0007$ & $0.035 \pm 0.058$ & -0.98 & $26.4 / 10$ \\
\hline & 6 & $0.1182 \pm 0.0001$ & $-0.293 \pm 0.010$ & 0.88 & $24.0 / 10$ \\
\hline \multirow[t]{5}{*}{$\left\langle(1-T)^{2}\right\rangle$} & 2 & $0.1463 \pm 0.0009$ & $1.855 \pm 0.455$ & 0.73 & $23.8 / 10$ \\
\hline & 3 & $0.1426 \pm 0.0009$ & $1.762 \pm 0.454$ & 0.74 & $24.0 / 10$ \\
\hline & 4 & $0.1416 \pm 0.0008$ & $1.644 \pm 0.458$ & 0.73 & $24.2 / 10$ \\
\hline & 5 & $0.1412 \pm 0.0008$ & $1.546 \pm 0.460$ & 0.73 & $24.3 / 10$ \\
\hline & 6 & $0.1410 \pm 0.0008$ & $1.465 \pm 0.470$ & 0.73 & $24.4 / 10$ \\
\hline \multirow[t]{5}{*}{$\left\langle(1-T)^{3}\right\rangle$} & 2 & $0.1523 \pm 0.0015$ & $0.273 \pm 0.136$ & 0.73 & $20.7 / 10$ \\
\hline & 3 & $0.1525 \pm 0.0015$ & $0.274 \pm 0.137$ & 0.73 & $20.7 / 10$ \\
\hline & 4 & $0.1531 \pm 0.0015$ & $0.286 \pm 0.137$ & 0.73 & $20.7 / 10$ \\
\hline & 5 & $0.1533 \pm 0.0015$ & $0.298 \pm 0.136$ & 0.73 & $20.6 / 10$ \\
\hline & 6 & $0.1534 \pm 0.0015$ & $0.307 \pm 0.135$ & 0.74 & $20.6 / 10$ \\
\hline \multirow[t]{5}{*}{$\left\langle(1-T)^{4}\right\rangle$} & 2 & $0.1581 \pm 0.0017$ & $0.059 \pm 0.024$ & 0.55 & $17.6 / 10$ \\
\hline & 3 & $0.1625 \pm 0.0018$ & $0.063 \pm 0.024$ & 0.55 & $17.5 / 10$ \\
\hline & 4 & $0.1645 \pm 0.0019$ & $0.075 \pm 0.023$ & 0.56 & $17.4 / 10$ \\
\hline & 5 & $0.1652 \pm 0.0019$ & $0.085 \pm 0.022$ & 0.58 & $17.3 / 10$ \\
\hline & 6 & $0.1655 \pm 0.0019$ & $0.095 \pm 0.021$ & 0.60 & $17.3 / 10$ \\
\hline
\end{tabular}

the experimental systematic uncertainty, the fit is repeated employing the minimum overlap assumption for the experimental uncertainties of the data. To estimate the theoretical uncertainty we study the following contributions:

- In order $\mathcal{O}\left(\bar{a}^{6}\right)$ of the perturbative prediction (27) the unknown coefficient $\beta_{4}$ appears. We set it to the simple Pade estimate $^{12} \beta_{4}=\beta_{3}^{2} / \beta_{2}$ instead of $\beta_{4}=0$. This does not change any of the four cited digits of the $\alpha_{\mathrm{S}}\left(M_{\mathrm{Z}^{0}}\right)$ fit result.

- Instead of three loops we use the four known loops for calculating the $Q$ evolution of $\alpha_{\mathrm{S}}$. This changes the fit result by less than 0.0001 .

- The $\mathcal{O}\left(\bar{a}^{2}\right)$ coefficient $B_{1}$ is approximated by SDG within $34 \%$ [10], and the approximation is expected to be complete in high order [9]. Thus we vary the coefficient $C_{1}$ by $+34 \%$ or $-34 \%$. This results in changes of $\alpha_{\mathrm{S}}\left(M_{\mathrm{Z}^{0}}\right)$ by -0.0027 or +0.0031 . This variation is consistent with truncating after expansion order $m_{\max }=3$ or $m_{\max }=6$. A renormalisation scale variation is not studied and is expected to cause a small effect in a prediction of fifth order.

- As $v_{1}$ is one of the two fit parameters, the hadronisation error is part of the fit error.

\footnotetext{
${ }^{12}$ In this approximation the coefficients $\beta_{i}$ follow a simple geometric series.
}

The result is

$$
\begin{aligned}
\alpha_{\mathrm{S}}\left(M_{Z^{0}}\right)= & 0.1172 \pm 0.0007 \text { (stat.) } \pm 0.0016(\text { exp. }) \\
& +0.0031 \text { (theo.) } \\
& -0.0027 \text { (tot.) }
\end{aligned}
$$

It is our most precise value. The variation of the NNLO coefficient $C_{1}$ by $\pm 34 \%$ is better motivated than the somewhat arbitrary variation of the renormalisation scale factor $x_{\mu}=0.5,2.0$. It leads to a smaller perturbative uncertainty than in the NLO analyses discussed above or in [5].

In [9] the SDG prediction was fitted to $\langle 1-T\rangle$ data, notably of the PETRA experiments TASSO and MARK $J$ at cms energies down to $12 \mathrm{GeV}$. The results were $\alpha_{\mathrm{S}}\left(M_{\mathrm{Z}^{0}}\right)=$ 0.110 and $\lambda_{1}=0.62 \mathrm{GeV}$. In the old PETRA measurements $\mathrm{e}^{+} \mathrm{e}^{-} \rightarrow \mathrm{b} \overline{\mathrm{b}}$ events have not been subtracted. They enlarge $\langle 1-T\rangle$ in an effect decreasing with energy faster than $1 / Q$. This results in an increase of $\lambda_{1}$ and a decrease of $\alpha_{\mathrm{S}}\left(M_{\mathrm{Z}^{0}}\right)$.

\section{Summary}

As an alternative to the description of hadronisation by Monte Carlo models we studied analytical models of hadronisation. These descriptions require no laborious tuning of 

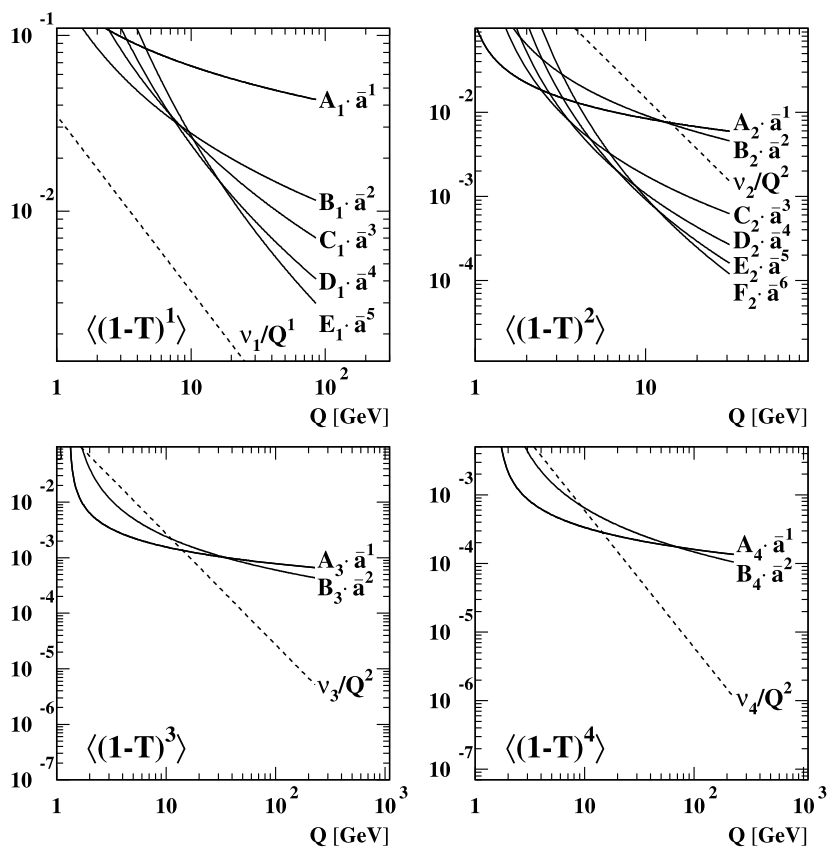

Fig. $9 Q$ evolution of the employed perturbative terms from (28) and of the leading power correction in powers of the cms energy $Q$ for the case of first to fourth moment of thrust. $A_{n} \ldots F_{n}$ are the coefficients of the terms in 1st... 6th order of the coupling $\bar{a}$ as defined in (26), (27). The parameters $\alpha_{\mathrm{S}}\left(M_{\mathrm{Z}^{0}}\right)$ and $v_{i}$ are fitted to the data

a Monte Carlo model but fitting of $\alpha_{S}$ and an additional parameter. In this way, their consistency can be quantified.

We studied the dispersive model, the shape function and the single dressed gluon approximation, including the NLO predictions. All models describe the mean values of all studied event shape variables at cms energies $Q=$ $14 \ldots 209 \mathrm{GeV}$ well. The dispersive model and the shape function also describe the higher moments of the one-hemisphere variables well. The measurements of $\alpha_{\mathrm{S}}\left(M_{\mathrm{Z}^{0}}\right)$ and $\alpha_{0}(2 \mathrm{GeV})$ in the dispersive model from the mean values of $1-T, C, B_{\mathrm{T}}$, all studied moments of $y_{23}^{\mathrm{D}}$ and $\left\langle M_{\mathrm{H}}^{2}\right\rangle,\left\langle M_{\mathrm{H}}^{4}\right\rangle$ confirm the universality of these parameters within total errors.

Higher moments of the two-hemisphere variables $1-T$, $B_{\mathrm{T}}$ and $C$ can only be described by higher values of $\alpha_{\mathrm{S}}\left(M_{\mathrm{Z}^{0}}\right)$, moments of $B_{\mathrm{W}}$ and $M_{\mathrm{H}}$ only by higher values of the power correction coefficient. Both deviations apparently compensate for the deficiencies of the perturbative predictions. The issue of high $\alpha_{\mathrm{S}}$ values for higher moments of the two-hemisphere variables is expected to be alleviated by the new perturbative NNLO predictions [19] that help to describe the multijet region better.

Averaging the parameters from $\left\langle(1-T)^{1}\right\rangle,\left\langle C^{1}\right\rangle,\left\langle B_{\mathrm{T}}^{1}\right\rangle$, $\left\langle\left(y_{23}^{\mathrm{D}}\right)^{1}\right\rangle \ldots\left\langle\left(y_{23}^{\mathrm{D}}\right)^{5}\right\rangle$, and $\left\langle M_{\mathrm{H}}^{2}\right\rangle,\left\langle M_{\mathrm{H}}^{4}\right\rangle$ in the dispersive model results in
$\alpha_{\mathrm{S}}\left(M_{\mathrm{Z}^{0}}\right)=0.1183 \pm 0.0056$ (tot.),

$\alpha_{0}(2 \mathrm{GeV})=0.493 \pm 0.058$ (tot.).

Event shape moments are barely sensitive to more strongly suppressed power corrections resulting from the shape function. In general, a detailed understanding of non-perturbative effects cannot reach any further than the reliability of the underlying perturbative structure which was studied in $[5,6]$.

For some variables the variance shows an unexpected energy evolution. Of the variables analysed here only the $1-T$ distributions are broader at low cms energy; the $M_{\mathrm{H}}^{2}, C, B_{\mathrm{T}}$ and $B_{\mathrm{W}}$ distributions are narrower at low energy. The energy evolution of the variances is not given correctly by the pure NLO prediction. The description by an additional power correction from the shape function is not quantitatively correct.

The single dressed gluon approximation shows the same problems describing the higher thrust moments as the other models. Fitting the prediction for $\left\langle(1-T)^{1}\right\rangle$ including terms up to fifth order gives power corrections compatible with zero and

$\alpha_{\mathrm{S}}\left(M_{\mathrm{Z}^{0}}\right)=0.1172 \pm 0.0036$ (tot.).

With a competitive total error of 3\% this is our most precise $\alpha_{\mathrm{S}}$ measurement. It agrees with the world average of $\alpha_{\mathrm{S}}\left(M_{\mathrm{Z}^{0}}\right)=0.1189 \pm 0.0010$ [45]. For this result a Monte Carlo model has only been used for correcting the data for $\mathrm{b} \bar{b}$ background, taking into account its uncertainty in the experimental systematic error.

Moments are an interesting alternative to distributions: Specific parts of phase space are tested selectively and the energy evolution shows up clearly. This evolution can be studied thoroughly by the combination of the experiments JADE and OPAL. As in [5] the most interesting energy range is provided by the JADE experiment, as the perturbative and non-perturbative effects both scale inversely with the energy.

Acknowledgements We would like to thank E. Gardi and G. Korchemsky for explanations of their work and useful discussions. This research was supported by the DFG cluster of excellence 'Origin and Structure of the Universe'.

\section{References}

1. H. Fritzsch, M. Gell-Mann, H. Leutwyler, Phys. Lett. B 47, 365 (1973)

2. D. Gross, F. Wilczek, Phys. Rev. Lett. 30, 1343 (1973)

3. D. Gross, F. Wilczek, Phys. Rev. D 8, 3633 (1973)

4. H. Politzer, Phys. Rev. Lett. 30, 1346 (1973)

5. C. Pahl, S. Bethke, S. Kluth, J. Schieck, Eur. Phys. J. C 60, 181 (2009)

6. G. Abbiendi et al. (OPAL Collaboration), Eur. Phys. J. C 40, 287 (2005)

7. Y. Dokshitzer, G. Marchesini, B. Webber, Nucl. Phys. B 469, 93 (1996)

8. G. Korchemsky, S. Tafat, J. High Energy Phys. 0010, 010 (2000)

9. E. Gardi, G. Grunberg, J. High Energy Phys. 9911, 016 (1999) 
10. E. Gardi, J. High Energy Phys. 0004, 030 (2000)

11. A. Heister et al. (ALEPH Collaboration), Eur. Phys. J. C 35, 457 (2004)

12. P. Achard et al. (L3 Collaboration), Phys. Rep. 399, 71 (2004)

13. P. Abreu et al. (DELPHI Collaboration), Phys. Lett. B 456, 322 (1999)

14. P.A. Movilla Fernández, S. Bethke, O. Biebel, S. Kluth et al. (JADE Collaboration), Eur. Phys. J. C 22, 1 (2001)

15. M. Dasgupta, G. Salam, J. Phys. G 30, R143 (2004)

16. A. Gehrmann-De Ridder, T. Gehrmann, E.W.N. Glover, G. Heinrich, J. High Energy Phys. 12, 094 (2007)

17. S. Weinzierl, Phys. Rev. Lett. 101, 162001 (2008)

18. P.A. Movilla Fernández, O. Biebel, S. Bethke, S. Kluth, P. Pfeifenschneider et al. (JADE Collaboration), Eur. Phys. J. C 1, 461 (1998)

19. A. Gehrmann-De Ridder, T. Gehrmann, E.W.N. Glover, G. Heinrich, J. High Energy Phys. 05, 106 (2009)

20. S. Catani, M. Seymour, Phys. Lett. B 378, 287 (1996)

21. R. Ellis, D. Ross, A. Terrano, Nucl. Phys. B 178, 421 (1981)

22. M. Beneke, Phys. Rep. 317, 1 (1999)

23. C. Pahl, in High Energy Physics ICHEP 2008, Proceedings of the 34th International Conference, to appear

24. Y. Dokshitzer, G. Marchesini, B. Webber, Phys. Lett. B 352, 451 (1995)

25. Y. Dokshitzer, in High Energy Physics ICHEP 1998, Proceedings of the 29th International Conference (1999), p. 305

26. R. Ellis, W. Stirling, B. Webber, QCD and Collider Physics. Cambridge Monographs on Particle Physics, Nuclear Physics and Cosmology, vol. 8 (Cambridge University Press, Cambridge, 1996)
27. Y. Dokshitzer, A. Lucenti, G. Marchesini, G. Salam, Nucl. Phys. B 511, 396 (1998)

28. Y. Dokshitzer, A. Lucenti, G. Marchesini, G. Salam, J. High Energy Phys. 05, 003 (1998)

29. M. Dasgupta, L. Magnea, G. Smye, J. High Energy Phys. 11, 025 (1999)

30. P.A. Movilla Fernández, Nucl. Phys. Proc. Suppl. 74, 384 (1999)

31. Y. Dokshitzer, G. Marchesini, G. Salam, Eur. Phys. J. C 1, 3 (1999)

32. O. Biebel, Phys. Rep. 340, 165 (2001)

33. B. Webber, Nucl. Phys. Proc. Suppl. 71, 66 (1999)

34. H.J. Lu, C.A.R. Sa de Melo, Phys. Lett. B 273, 260 (1991)

35. E. Gardi, Private communication

36. T. van Ritbergen, J. Vermaseren, S. Larin, Phys. Lett. B 400, 379 (1997)

37. M. Czakon, Nucl. Phys. B 710, 485 (2005)

38. P. Pfeifenschneider et al. (JADE and OPAL Collaboration), Eur. Phys. J. C 17, 19 (2000)

39. P.A. Movilla Fernández, Ph.D. thesis, RWTH Aachen (2003). http://nbn-resolving.de/urn:nbn:de:hbz:82-opus-4836

40. S. Kluth, Rep. Prog. Phys. 69, 1771 (2006)

41. G. Salam, D. Wicke, J. High Energy Phys. 05, 061 (2001)

42. Delphi collaboration, 2000-116 CONF415 (2000), unpublished

43. http://durpdg.dur.ac.uk/HEPDATA/

44. C. Pahl, Ph.D. thesis, TU München (2007). http://nbn-resolving. de/urn:nbn:de:bvb:91-diss-20070906-627360-1-2

45. S. Bethke, Prog. Part. Nucl. Phys. 58, 351 (2006) 